\title{
POTTS MODELS AND RANDOM-CLUSTER PROCESSES WITH MANY-BODY INTERACTIONS
}

\author{
GeOFFrey GRIMMEtT
}

\begin{abstract}
Known differential inequalities for certain ferromagnetic Potts models with pairinteractions may be extended to Potts models with many-body interactions. As a major application of such differential inequalities, we obtain necessary and sufficient conditions on the set of interactions of such a Potts model in order that its critical point be a strictly monotonic function of the strengths of interactions. The method yields some ancillary information concerning the equality of certain critical exponents for Potts models; this amounts to a small amount of rigorous universality. These results are achieved in the context of a 'Fortuin-Kasteleyn representation' of Potts models with many-body interactions. For such a Potts model, the corresponding random-cluster process is a (random) hypergraph.
\end{abstract}

\section{Introduction}

In the study of phase transition, it is commonly the case that the value of the critical point is a monotonic function of the strengths of local interactions. For example, the critical temperature of a ferromagnetic Ising model cannot decrease if the intensity of any pairinteraction is increased. Such (weak) monotonicity, when true, is usually easy to prove. Strict monotonicity, on the other hand, presents special difficulties. A general approach to the problem of proving strict monotonicity of the critical point was described by Aizenman and Grimmett (1991); they obtained necessary and sufficient conditions for strict monotonicity in percolation, and in addition proved strict monotonicity for certain Ising models with pair-interactions. The corresponding results for Potts models and randomcluster processes have been established by Bezuidenhout, Grimmett, and Kesten (1993) (subsequently referred to as $[\mathrm{BGK}]$ ), for processes with pair-interactions. Since the Ising model is an instance of a Potts model, the latter work generalizes some of the conclusions of Aizenman and Grimmett (1991). It is our purpose in this paper to go further than $[\mathrm{BGK}]$, and to show how strict monotonicity may be established for processes with many-body interactions. The basic techniques developed in [BGK] are central to the arguments presented here, but the present paper is self-contained with only little overlap of mathematical content with [BGK]. Further discussion is provided in Grimmett (1993).

Rather than working directly with Potts models, we shall work with the corresponding random-cluster processes (sometimes known as Fortuin-Kasteleyn processes). In the case of pair-interactions, the relationship between Potts and random-cluster processes is well understood (see Aizenman, Chayes, Chayes, and Newman (1988), Edwards and Sokal

1991 Mathematics Subject Classification. 60K35, 82B20.

Key words and phrases. Potts model, Ising model, random-cluster process, critical temperature, critical exponent, differential inequality, universality. 
(1988), Grimmett (1994), and the references therein). Potts models with many-body interactions do not appear to have been studied much in this regard, but it is straightforward to see that they correspond to random-cluster processes which are hypergraphs rather than the more usual graphs (i.e., the incidence relation is not that of pairs of vertices forming 'edges', but of finite sets of vertices forming 'hyperedges'). Furthermore, the magnetization of such a Potts model corresponds to the probability of certain infinite paths in the random-cluster process. This correspondence enables one to study the Potts phase transition by way of the random-cluster process.

In Section 2, we sketch the relationship between Potts models and random-cluster processes, in the presence of many-body interactions. The class of random-cluster measures is (in a certain sense) more general than the subclass of such measures arising from Potts models, and it is in this more general context that the results of this paper will be stated and proved.

In advance of sketching the main results, we make some remarks about the models studied. The simplest $d$-dimensional systems inhabit the hypercubic lattice $\mathbb{Z}^{d}$, with edges joining all pairs of points which are (euclidean) distance 1 apart. Commonly each edge has an associated interaction $J$, which may be constant for all edges. Greater generality may be obtained by one or more of the following constructions:

(a) allow different edges to have different values of $J$,

(b) allow a different set of edges,

(c) allow many-body interactions, rather than pair-interactions only.

Authors often establish results for the simplest case above, and then may argue loosely that similar results may be obtained for more general systems. The truth of such an assertion is sometimes far from evident. When studying such systems in generality, new difficulties usually arise. In the context of strict inequalities, no earlier author has obtained general results, and it is desirable to establish beyond doubt that general results are indeed valid; see Hammersley (1961), Kesten (1982, Chap. 10), Menshikov (1987), Aizenman and Grimmett (1991), [BGK]. (Certain results for percolation have also been found contemporaneously by Wierman (1993).) In the present context, we obtain a necessary and sufficient condition on the interactions of a Potts model in order that the critical point is strictly monotone in the interactions; see Theorem 3. This condition has a novel structure which is not evident from the easier case of pair-interactions on $\mathbb{Z}^{d}$ (i.e., the concept of an 'essential' interaction introduced in Section 3). Various graph-theoretic difficulties arise in proving this result, and we see no way of avoiding the lengthy technicalities laid out later in this paper.

Let $q \in[1, \infty)$, and let $\mathbf{p}=\left(p_{e}: e \in K\right)$ be a vector of real numbers satisfying $0 \leq p_{e} \leq 1$ and indexed by a finite family $K$ of finite subsets of $\mathbb{Z}^{d}$. There exists (as described in Section 3) a random-cluster measure $\phi_{\mathbf{p}, q}$ on the set of hypergraphs having $\mathbb{Z}^{d}$ as vertex set, and having as set $\mathbb{E}$ of hyperedges the union of certain periodic families of translates of the sets in $K$. Thus the family $K$ contains a list of the basic interacting sets of the measure, and these sets describe a pattern which is repeated elsewhere in the lattice by periodic translations. The 'order parameter' of the measure $\phi_{\mathbf{p}, q}$ is the function

$$
\theta(\mathbf{p}, q)=\phi_{\mathbf{p}, q}(0 \leftrightarrow \infty),
$$

the probability that the origin of $\mathbb{Z}^{d}$ is in an infinite component of a random hypergraph sampled according to the measure. Since $q \geq 1$, it is the case that the measure $\phi_{\mathbf{p}, q}$ satisfies the FKG inequality; consequently, $\theta(\cdot, q)$ is non-decreasing in $\mathbf{p}$ (see Aizenman et al. (1988, p. 9)). The zero-set of the function $\theta(\cdot, q)$ gives rise to a 'critical surface' $\mathrm{C}_{q}$, being the 
set of vectors $\mathbf{p}$ such that all open neighbourhoods of $\mathbf{p}$ (in $\mathbb{R}^{K}$ ) contain points $\mathbf{p}^{\prime}$ with $\theta\left(\mathbf{p}^{\prime}, q\right)=0$ as well as points $\mathbf{p}^{\prime \prime}$ with $\theta\left(\mathbf{p}^{\prime \prime}, q\right)>0$.

We address the problem of whether or not $\mathrm{C}_{q}$ has 'flat sections' in the sense that there exist $\mathbf{p}, \mathbf{p}^{\prime} \in \mathrm{C}_{q}$ with $\mathbf{p} \leq \mathbf{p}^{\prime}$ but $\mathbf{p} \neq \mathbf{p}^{\prime}$ (all vector inequalities in this paper of the form $\mathbf{x} \leq \mathbf{y}$ should be interpreted coordinatewise). It turns out that, when the problem is suitably formulated, the set $K$ of generating elements may be partitioned as $K=\mathcal{E} \cup \mathcal{I}$ into a set $\mathcal{E}$ of 'essential' elements and a set $\mathcal{I}$ of 'inessential' elements, according to the following property. Let $e \in K, \mathbf{p} \in \mathrm{C}_{q}$, and let $\mathbf{p}^{\prime} \in(0,1)^{K}$ be a vector satisfying $p_{e} \neq p_{e}^{\prime}$, $p_{f}=p_{f}^{\prime}$ for all $f \neq e$; thus $\mathbf{p}$ and $\mathbf{p}^{\prime}$ differ only in the component indexed by $e$. Then

$$
\mathbf{p}^{\prime} \begin{cases}\notin \mathrm{C}_{q} & \text { if } e \in \mathcal{E} \\ \in \mathrm{C}_{q} & \text { if } e \in \mathcal{I} .\end{cases}
$$

That is to say, the value of $p_{e}$ (for $e \in \mathcal{I}$ ) is immaterial to whether or not $\mathbf{p} \in \mathrm{C}_{q}$, whereas the value of $p_{e}$ (for $e \in \mathcal{E}$ ) is highly relevant. In other words, certain elements of $K$ contribute in an essential way to the phase transition, whilst others make no vital contribution. We present, in Section 3, a characterization of the essential elements (i.e., the elements in $\mathcal{E}$ ) as those elements of $K$ which contain the endvertices of two vertexdisjoint infinite paths in the hypergraph $\left(\mathbb{Z}^{d}, \mathbb{E}\right)$; actually we shall take the last statement as a definition of the elements of $\mathcal{E}$, and shall prove (1.2). Theorems 1-3, in Section 3, are the main results of the paper.

Throughout, we make use of the methodology developed by Menshikov (1987), Aizenman and Grimmett (1991), and [BGK]. The basic approach is to prove inequalities for the partial derivatives of the finite-box approximation $\theta_{\Lambda}$ to the function $\theta$. Let $\Lambda$ be a finite box, let $\phi_{\Lambda, \mathbf{p}, q}$ be the random-cluster measure on $\Lambda$ with parameters $\mathbf{p}$ and $q$ (with 'wired' boundary conditions), and let $\theta_{\Lambda}(\mathbf{p}, q)$ be the $\phi_{\Lambda, \mathbf{p}, q}$-probability that the origin is joined to the boundary of $\Lambda$. We shall prove that there exists a continuous function $\alpha:(0,1)^{K} \rightarrow(0, \infty)$ such that

$$
\frac{\partial \theta_{\Lambda}}{\partial p_{e}} \leq \alpha(\mathbf{p}) \frac{\partial \theta_{\Lambda}}{\partial p_{f}} \quad \text { for all } \mathbf{p} \in(0,1)^{K}, \text { and } e, f \in \mathcal{E},
$$

and for all sufficiently large $\Lambda$. Taken in conjunction with a proof that elements in $\mathcal{I}$ $(=K \backslash \mathcal{E})$ do not contribute to the phase transition, inequality (1.3) turns out to be enough to imply (1.2). We shall prove (1.3) by the route laid down in [BGK]; the (manybody) generality of the current paper leads to certain difficulties not encountered in [BGK], and these are resolved in Sections 5 and 6 by (hyper)graph-theoretic arguments.

As remarked in [BGK], the differential inequalities (1.3) have implications for the values of certain critical exponents, particularly those which describe the behaviour of the function $\theta(\cdot, q)$ at points close to $\mathrm{C}_{q}$. Let $\mathbf{p} \in \mathrm{C}_{q}$ and let $\mathbf{u}$ lie in the (euclidean) unit sphere $V$ of $\mathbb{R}^{K}$. Let

$$
\beta_{\mathbf{p}}(\mathbf{u})=\lim _{\epsilon \downarrow 0}\left\{\frac{\log [\theta(\mathbf{p}+\epsilon \mathbf{u}, q)-\theta(\mathbf{p}, q)]}{\log \epsilon}\right\}
$$

if this limit exists. Subject to the existence of such exponents, inequality (1.3) may be used to show that, for given $\mathbf{p} \in \mathrm{C}_{q}$, the value of $\beta_{\mathbf{p}}(\mathbf{u})$ is constant for all vectors $\mathbf{u}$ lying in 
some open subset of $V$ which contains the 'axial' unit vectors $\mathbf{u}_{e}=\left(\delta_{e f}: f \in K\right)$ for $e \in \mathcal{E}$, where $\delta_{e f}$ is the Kronecker delta. The argument of [BGK] is valid in this regard with almost no change, with the forthcoming Theorem 1 as a starting point; we shall therefore omit further details, referring the reader to the earlier paper and to the example at the end of this section, beginning just before (1.7).

We terminate this section with simple examples of the type of results derived in this paper. Let $q$ be an integer satisfying $q \geq 2$, and write $\Sigma=\{1,2, \ldots, q\} \mathbb{Z}^{2}$; we think of $\sigma$ $(\in \Sigma)$ as an allocation of a spin $\sigma(y)$ to each vertex $y\left(\in \mathbb{Z}^{2}\right)$, where $\sigma(y) \in\{1,2, \ldots, q\}$. Let $\Sigma_{\Lambda}$ be the set of all elements $\sigma(\in \Sigma)$ which satisfy $\sigma(y)=1$ if $y \notin \Lambda \backslash \partial \Lambda$, where $\Lambda$ is some finite box of $\mathbb{Z}^{2}$ and $\partial \Lambda$ is the boundary of $\Lambda$. We wish to consider a Potts measure on $\Sigma$, whose interactions are possibly many-body, and we give two examples (see Figure $1)$.

Example 1. Let $e_{1}=\{0,1,2\}^{2}$, a square with side-length 2, and let $e_{2}=\{0,1\} \times\{1\}$, an edge with endpoints $(0,1)$ and $(1,1)$. The sets $e_{i}$ are the basic interacting bodies of the process, and we consider all translations of $e_{1}$ and $e_{2}$ by multiples of the vector $(2,2)$; i.e., we denote by $\mathbb{E}$ the set $\mathbb{E}=\mathbb{E}_{1} \cup \mathbb{E}_{2}$, where

$$
\mathbb{E}_{i}=\left\{e_{i}+\left(2 z_{1}, 2 z_{2}\right): z=\left(z_{1}, z_{2}\right) \in \mathbb{Z}^{2}\right\}
$$

Let $\mathbf{J}=\left(J_{1}, J_{2}\right)$ where $J_{1}, J_{2}>0$, and let

$$
H_{\Lambda}(\sigma)=\sum_{g \in \mathbb{E}_{\Lambda}} J_{g}\left(1-\delta_{\sigma}(g)\right), \quad \sigma \in \Sigma_{\Lambda}
$$

(this is a convenient way of defining the hamiltonian for what follows); here, $\mathbb{E}_{\Lambda}$ is the set of all members of $\mathbb{E}$ which are subsets of $\Lambda, J_{g}=J_{i}$ if $g$ is a translate of $e_{i}$, and

$$
\delta_{\sigma}(g)= \begin{cases}1 & \text { if } \sigma(u)=\sigma(v) \text { for all } u, v \in g \\ 0 & \text { otherwise }\end{cases}
$$

We then consider weak limits as $\Lambda \uparrow \mathbb{Z}^{2}$ of the Potts probability measure

$$
\pi_{\Lambda, \mathbf{J}, q}(\sigma)=\frac{1}{Z_{\Lambda}} \exp \left(-\beta H_{\Lambda}(\sigma)\right), \quad \sigma \in \Sigma_{\Lambda}
$$

where $\beta>0$ and $Z_{\Lambda}$ is the appropriate normalizing factor. There is a standard definition of the critical inverse-temperature $\beta_{c}(\mathbf{J}, q)$ for this model (see Section 3). A special case of the forthcoming Theorem 3 is that $\beta_{c}(\mathbf{J}, q)$ is strictly decreasing in $\mathbf{J}$, i.e., $\beta_{c}(\mathbf{J}, q)>\beta_{c}\left(\mathbf{J}^{\prime}, q\right)$ if $\mathbf{J} \leq \mathbf{J}^{\prime}$ but $\mathbf{J} \neq \mathbf{J}^{\prime}$. This conclusion is in contrast to that of the next (similar) example. Example 2. This differs from Example 1 in the single regard that $e_{1}$ and $e_{2}$ are replaced by $f_{1}=e_{1} \backslash\{1,1\}, f_{2}=e_{2}$. In this case $\beta_{c}(\mathbf{J}, q)$ is strictly decreasing in $J_{1}$ but is independent of $J_{2}$.

These two examples are very similar in form, but the corresponding conclusions are strikingly different. This difference arises in the following way. Any collection $\mathbb{E}$ of subsets of $\mathbb{Z}^{2}$ may be used to generate a graph having vertex set $\mathbb{Z}^{2}$ and edge set given by the adjacency relation

$$
u \sim v \text { if }\{u, v\} \subseteq g \text { for some } g \in \mathbb{E} .
$$


Let $G_{1}$ and $G_{2}$ be the two graphs thus generated in Examples 1 and 2 respectively (see Figure 1). A subset $A$ of $\mathbb{Z}^{2}$ is said to be '2-connected to $\infty$ in $G_{i}$ ' if there exist two vertex-disjoint infinite paths of $G_{i}$, each having its (unique) endpoint lying in $A$. It is easy to see from Figure 1 that both $e_{1}$ and $e_{2}$ are 2-connected to $\infty$ in $G_{1}$, and it is this graph-theoretic observation which leads to the conclusion that $\beta_{c}(\mathbf{J}, q)$ is strictly monotone in both $J_{1}$ and $J_{2}$. On the other hand, in $G_{2}, f_{1}$ is 2 -connected to $\infty$ but $f_{2}$ is not; as a consequence $\beta_{c}(\mathbf{J}, q)$ is strictly monotone in $J_{1}$ but not in $J_{2}$ in this case.

More generally, consider the vertex set $\mathbb{Z}^{d}$ (where $d \geq 2$ ) together with some finite collection $L$ of finite subsets of $\mathbb{Z}^{d}$ (in each of the above examples, $L$ contains exactly two sets); each set $e(\in L)$ generates a periodic family of translates of $e$. There is a family $\mathbf{J}=\left(J_{e}: e \in L\right)$ of parameters. It turns out that the critical point $\beta_{c}(\mathbf{J}, q)$ is a strictly monotonic function of a given parameter $J_{e}$ if and only if the corresponding set $e$ is 2connected to $\infty$ in the appropriate graph. (The relationship between the collection $L$ and the collection $K$ introduced before (1.1) will become clear soon.)

Such results will be obtained in the context of the random-cluster process with parameter $q$ satisfying $q \in(1, \infty)$. It is interesting to note that the arguments of this paper fail in the case $q=1$, i.e., the case of percolation. In the case of Example 1, the percolation process in question is that having the product measure on the space $\Omega_{\Lambda}=\{0,1\}^{\mathbb{E}_{\Lambda}}$ given by

$$
\nu_{\Lambda, \mathbf{p}}(\omega)=\prod_{g \in \mathbb{E}_{\Lambda}} p_{g}^{\omega(g)}\left(1-p_{g}\right)^{1-\omega(g)}, \quad \omega \in \Omega_{\Lambda},
$$

where $\mathbf{p}=\left(p_{1}, p_{2}\right) \in[0,1]^{2}$, and $p_{g}=p_{i}$ if $g$ is a translate of $e_{i}$. There is a standard definition of the critical surface of such a process (see Section 3). The arguments of Aizenman and Grimmett (1991) may be adapted to this general situation (as in the present paper) in order to obtain a condition on an interaction-set $e$ which is necessary and sufficient for the associated parameter $p_{e}$ to contribute to the phase transition in an essential way; for further discussion, see the second paragraph after Theorem 1 in Section 3.

Finally we illustrate our conclusions concerning critical exponents, making use of Example 1. Let $M(\mathbf{J}, \beta, q)$ denote the magnetization of the Potts model observed in the limit as $\Lambda \uparrow \mathbb{Z}^{2}$ of the measure given in (1.5), and let $\beta_{c}=\beta_{c}(\mathbf{J}, q)$ denote the critical point, for a given $\mathbf{J}$. With $\mathbf{J}=\left(J_{1}, J_{2}\right)$ fixed and $J_{1}, J_{2}>0$, we define the critical exponent

$$
\kappa_{\mathbf{J}}(\theta)=\lim _{\epsilon \downarrow 0}\left\{\frac{\log \left[M\left(\mathbf{J}(\theta, \epsilon), \beta_{c}, q\right)-M\left(\mathbf{J}, \beta_{c}, q\right)\right]}{\log \epsilon}\right\},
$$

where

$$
\mathbf{J}(\theta, \epsilon)=\left(J_{1}+\epsilon \cos \theta, J_{2}+\epsilon \sin \theta\right),
$$

for any angle $\theta$ for which $\kappa_{\mathbf{J}}(\theta)$ exists. (Such an exponent is usually denoted by $\beta$.) It is a consequence of Theorem $1(\mathrm{~b})$ that the value of $\kappa_{\mathbf{J}}(\theta)$ is constant for all $\theta$ satisfying

$$
-\theta_{1}<\theta<\frac{1}{2} \pi+\theta_{1}
$$

for some $\theta_{1}=\theta_{1}(\mathbf{J})>0$. That is to say, to a certain extent, the asymptotic behaviour of $M\left(\mathbf{J}^{\prime}, \beta_{c}, q\right)$, in the limit as $\mathbf{J}^{\prime} \rightarrow \mathbf{J}$, does not depend on the direction of approach by $\mathbf{J}^{\prime}$ 
of $\mathbf{J}$. In particular, there is a constant critical exponent whenever $\mathbf{J}^{\prime} \downarrow \mathbf{J}$, whatever the direction of this limit.

A corresponding conclusion is valid for any Potts or random-cluster process (with $q>1$ ) in any dimension, with possibly many-body interactions. Similarly, a corresponding result is valid for percolation processes (by dint of the discussion appearing above, and after Theorem 1). Wierman (1993) has reached related but more limited conclusions for certain percolation processes.

Above (1.7) we required that $J_{1}, J_{2}>0$, but actually this requirement may be weakened to obtain an interesting conclusion. If $J_{2}=0$, then there is only one type of interaction (i.e., the translates of $e_{1}$, being the sets in $\mathbb{E}_{1}$ ). The above observation concerning the equality of critical exponents is valid also at the point $\mathbf{J}=\left(J_{1}, 0\right)\left(\in \mathrm{C}_{q}\right)$; that is to say, there exists $\theta_{1}=\theta_{1}\left(J_{1}\right)>0$ such that $\kappa_{\mathbf{J}}(\theta)$ is constant for all $\theta$ satisfying $0 \leq \theta<\frac{1}{2} \pi+\theta_{1}$. In the case $\theta=0$, the exponent $\kappa_{\mathbf{J}}(0)$ is associated with a process of 'pure $e_{1}$ type', since $J_{2}$ is held equal to 0 . On the other hand, in the case $\theta=\frac{1}{2} \pi, \kappa_{\mathbf{J}}\left(\frac{1}{2} \pi\right)$ is associated with a marginal increase in the strength of interaction of sets in $\mathbb{E}_{2}$, being the translates of $e_{2}$. We obtain therefore a small amount of rigorous universality involving processes of different (but related) types. Such an observation is valid in general, subject to a graph-theoretic condition on the extra interaction (i.e., $e_{2}$ in the example above). We state this extra condition here, using the terminology and notation to be introduced later in Section 3. Let $e \in L$. Let $\mathbb{H}_{e}$ be the hypergraph obtained from $\mathbb{H}$ by deleting all hyperedges of the form $(e, z)$ with $z \in \mathbb{Z}^{d}$. We call e acceptable if $\mathbb{H}_{e}$ satisfies (3.2) and (3.3), and in addition there exist two infinite vertex-disjoint paths of $\mathbb{H}_{e}$ each containing some point of $e$. It is not difficult to see that $e_{2}$ is acceptable, in Example 1, whereas $e_{1}$ is not acceptable. It is precisely the property of acceptability which is relevant in the discussion earlier in this paragraph. See also the final paragraph of Section 4.

\section{Processes with many-body interactions}

The random-cluster representation for a Potts model with pair-interactions has been well documented by Aizenman et al. (1988), Edwards and Sokal (1988), Grimmett (1994), and others. A similar representation is valid for Potts models with many-body interactions, the corresponding random-cluster process being a hypergraph rather than an ordinary graph. Such a consideration of many-body interactions leads to a theory which is a generalization of the original work of Fortuin and Kasteleyn. In particular, the generalized random-cluster process studied here has broadly similar properties to those of the Fortuin-Kasteleyn (FK) representation for processes with pair-interactions, such as the FKG inequality and the comparison inequalities. We sketch this material in this section, beginning with some notation for hypergraphs.

Let $V$ be a (finite or infinite) set of vertices, and let $E$ be a family of subsets of $V$ each having cardinality at least 2. Members of $E$ are called hyperedges, and the pair $H=(V, E)$ is a hypergraph; see Berge (1973) for a general account of the theory of hypergraphs. It is convenient (for a notational reason which may be clear later) to permit multiple hyperedges, in the sense that any given subset of $V$ may occur more than once in $E$. We say that distinct vertices $u, v$ are adjacent to one another if there exists $e \in E$ such that $\{u, v\} \subseteq e$; if this holds, we write $u \sim v$. A path in $H$ is an alternating sequence $v_{1}, e_{1}, v_{2}, e_{2}, \ldots, e_{n}, v_{n+1}, \ldots$ of distinct vertices $v_{i}$ and distinct hyperedges $e_{j}$ such that $\left\{v_{i}, v_{i+1}\right\} \subseteq e_{i}$ for all $i \geq 1$. If the path contains only finitely many vertices, and terminates at $v_{n+1}$, it is said to connect $v_{1}$ to $v_{n+1}$ and to be of length $n(\geq 0)$. If 
the path contains infinitely many vertices, it is called infinite and is said to connect $v_{1}$ to $\infty$. The requirement that the hyperedges of a path be distinct is not essential for that which follows, but is convenient; in the case of conventional graph theory, the edges are inevitably distinct if the vertices are distinct.

For $A, B \subseteq V$, we write $A \stackrel{H}{\leftrightarrow} B$ if either $A \cap B \neq \varnothing$ or there exist $a \in A$ and $b \in B$ such that $a$ is connected to $b$ by a path in $H$. It is easily seen that $\stackrel{H}{\leftrightarrow}$ is an equivalence relation on $V$. The equivalence classes of this relation are called the components of $H$. A hypergraph is connected if it has a unique component.

Next we define Potts models and random-cluster processes associated with the hypergraph $H$. In doing so, we make use of the observation, due to Edwards and Sokal (1988), that such processes may be defined on the same probability space in a certain useful way. Suppose that the vertex set $V$ is finite. Let $q$ be an integer satisfying $q \geq 2$, and write $\Sigma_{V}=\{1,2, \ldots, q\}^{V}$, the set of 'spin vectors' $\sigma=(\sigma(y): y \in V)$ with $\sigma(y) \in\{1,2, \ldots, q\}$ for each vertex $y$. Let $\Omega_{E}=\{0,1\}^{E}$ be the set of 'hyperedgeconfigurations' $\omega=(\omega(e): e \in E)$ with $\omega(e) \in\{0,1\}$ for each hyperedge $e$. Let $\mathbf{p}=\left(p_{e}: e \in E\right)$ be a real vector satisfying $0 \leq p_{e} \leq 1$ for all $e$. We define a probability measure on $\Sigma_{V} \times \Omega_{E}$, having parameters $\mathbf{p}$ and $q$, by

$$
\mu_{H, \mathbf{p}, q}(\sigma, \omega)=\frac{1}{Z} \prod_{e \in E}\left\{\left(1-p_{e}\right) \delta_{\omega(e), 0}+p_{e} \delta_{\omega(e), 1} \delta_{\sigma}(e)\right\}, \quad \sigma \in \Sigma_{V}, \omega \in \Omega_{E},
$$

where $Z$ is the appropriate normalizing factor, $\delta_{i, j}$ is the Kronecker delta, and $\delta .(e): \Sigma_{V} \rightarrow$ $\{0,1\}$ is given by

$$
\delta_{\sigma}(e)= \begin{cases}1 & \text { if } \sigma(u)=\sigma(v) \text { for all } u, v \in e \\ 0 & \text { otherwise }\end{cases}
$$

Following elementary calculations (as in Edwards and Sokal (1988)), one obtains that the marginal measures of $\mu_{H, \mathbf{p}, q}$ are

$$
\begin{aligned}
& \pi_{H, \mathbf{p}, q}(\sigma)=\frac{1}{Z} \prod_{e \in E}\left\{\left(1-p_{e}\right)+p_{e} \delta_{\sigma}(e)\right\}, \quad \sigma \in \Sigma_{V}, \\
& \phi_{H, \mathbf{p}, q}(\omega)=\frac{1}{Z}\left\{\prod_{e \in E} p_{e}^{\omega(e)}\left(1-p_{e}\right)^{1-\omega(e)}\right\} q^{k(\omega)}, \quad \omega \in \Omega_{E} ;
\end{aligned}
$$

here, $k(\omega)$ is the number of components of the hypergraph $(V, \eta(\omega))$ where $\eta(\omega)=\{e \in$ $E: \omega(e)=1\}$ is the set of 'open hyperedges' of $\omega$. The measures $\pi_{H, \mathbf{p}, q}$ and $\phi_{H, \mathbf{p}, q}$ are the well-known Potts and random-cluster measures, but in the context of many-body interactions. This is seen in the first case by rewriting $(2.2)$ in the form

$$
\pi_{H, \mathbf{p}, q}(\sigma)=\frac{1}{Z} \prod_{e \in E} \exp \left(-\beta J_{e}\left\{1-\delta_{\sigma}(e)\right\}\right), \quad \sigma \in \Sigma_{V},
$$

where the parameters $\beta$ and $\mathbf{J}=\left(J_{e}: e \in E\right)$ satisfy $\beta>0$ and

$$
e^{-\beta J_{e}}=1-p_{e}, \quad e \in E .
$$

The quantity $\beta$ is the 'inverse temperature' and the $J_{e}$ are the ferromagnetic coupling variables. 
The conditional measure $\mu_{H, \mathbf{p}, q}(\sigma \mid \omega)$ is given as follows. Let $\omega \in \Omega_{E}$. By (2.1), whenever $e$ is such that $\omega(e)=0$, then no constraint is placed on the values of $\sigma$ in consequence of this fact. On the other hand, if $e$ is such that $\omega(e)=1$, then there is (conditional) probability 1 that $\delta_{\sigma}(e)=1$, which is to say that the spins of all vertices in $e$ are equal. There being no further constraints (conditional on $\omega$ ), we deduce that there is equal (conditional) probability on any spin vector which has the property of being constant on each component of $(V, \eta(\omega))$. That is to say, to each of the $k(\omega)$ components of $(V, \eta(\omega))$ is allocated a random spin, such spins being uniform on $\{1,2, \ldots, q\}$ and independent of each other. There are $q^{k(\omega)}$ such possible spin vectors, and therefore

$$
\mu_{H, \mathbf{p}, q}(\sigma \mid \omega)= \begin{cases}q^{-k(\omega)} & \text { if } \sigma(u)=\sigma(v) \text { whenever } u \leftrightarrow v \\ 0 & \text { otherwise, }\end{cases}
$$

where $A \leftrightarrow B$ means that there exists $a \in A(\subseteq V)$ and $b \in B(\subseteq V)$ such that $a$ and $b$ are in the same component of the random hypergraph $(V, \eta(\omega))$.

For $u, v \in V$, let

$$
\tau_{H, \mathbf{p}, q}(u, v)=\sum_{\sigma \in \Sigma_{V}}\left(\delta_{\sigma(u), \sigma(v)}-q^{-1}\right) \pi_{H, \mathbf{p}, q}(\sigma),
$$

the 'two-point correlation function' of the Potts measure given in (2.2). Using the fact that $\pi_{H, \mathbf{p}, q}$ is a marginal of $\mu_{H, \mathbf{p}, q}$, we obtain that

$$
\begin{aligned}
\tau_{H, \mathbf{p}, q}(u, v) & =\sum_{\sigma, \omega}\left(\delta_{\sigma(u), \sigma(v)}-q^{-1}\right) \mu_{H, \mathbf{p}, q}(\sigma, \omega) \\
& =\sum_{\omega \in \Omega_{E}} \phi_{H, \mathbf{p}, q}(\omega)\left\{\sum_{\sigma \in \Sigma_{V}}\left(\delta_{\sigma(u), \sigma(v)}-q^{-1}\right) \mu_{H, \mathbf{p}, q}(\sigma \mid \omega)\right\} \\
& =\sum_{\omega \in \Omega_{E}} \phi_{H, \mathbf{p}, q}(\omega)\left\{\left(1-q^{-1}\right) I_{\{u \leftrightarrow v\}}(\omega)\right\} \\
& =\left(1-q^{-1}\right) \phi_{H, \mathbf{p}, q}(u \leftrightarrow v),
\end{aligned}
$$

where $I_{F}$ denotes the indicator function of an event $F\left(\subseteq \Omega_{E}\right)$. It is a consequence of (2.7) that long-range order in the Potts model corresponds exactly to the existence of long paths in the random-cluster process.

Having sketched the relationship between Potts and random-cluster measures, we turn our attention to the latter, given in (2.3); we make four notes concerning such measures.

(a) The parameter $q$. The measure $\phi_{H, \mathbf{p}, q}$ is well defined for all positive real values of $q$, not merely the integers; henceforth, when studying random-cluster measures, we shall assume that $q \in(0, \infty)$.

(b) The FKG inequality. There is a partial order on $\Omega_{E}$ given by $\omega \leq \omega^{\prime}$ if and only if $\omega(e) \leq \omega^{\prime}(e)$ for all $e \in E$. A function $f: \Omega_{E} \rightarrow \mathbb{R}$ is called increasing if $f(\omega) \leq f\left(\omega^{\prime}\right)$ whenever $\omega \leq \omega^{\prime}$. An event $F \subseteq \Omega_{E}$ is called increasing if its indicator function $I_{F}$ is increasing. Having settled the necessary terminology, we point out that the measure $\phi_{H, \mathbf{p}, q}$ satisfies the FKG inequality so long as $q \geq 1$ :

$$
\phi_{H, \mathbf{p}, q}(F \cap G) \geq \phi_{H, \mathbf{p}, q}(F) \phi_{H, \mathbf{p}, q}(G)
$$


for all increasing events $F$ and $G$, if $q \geq 1$ (similarly, increasing integrable random variables are positively correlated). The proof of this follows that of Aizenman et al. (1988, Thm 2.2 ), with one change in their equation (2.18) arising from the fact that

$$
k\left(\omega^{e}\right)-k\left(\omega_{e}\right)=1-k_{e}\left(\omega_{e}\right), \quad \text { for } e \in E,
$$

where $\omega^{e}$ and $\omega_{e}$ are the hyperedge-configurations given by

$$
\omega^{e}(f)=\left\{\begin{array}{ll}
\omega(f) & \text { if } f \neq e, \\
1 & \text { if } f=e,
\end{array} \quad \omega_{e}(f)= \begin{cases}\omega(f) & \text { if } f \neq e, \\
0 & \text { if } f=e,\end{cases}\right.
$$

and $k_{e}\left(\omega^{\prime}\right)$ is the number of components of the hypergraph $\left(V, \eta\left(\omega^{\prime}\right)\right)$ which contain vertices belonging to the hyperedge $e$.

(c) Comparison inequalities. Given two measures $\phi_{1}, \phi_{2}$ on $\Omega_{E}$, we write $\phi_{1} \leq \phi_{2}$ if

$$
\sum_{\omega \in \Omega_{E}} f(\omega) \phi_{1}(\omega) \leq \sum_{\omega \in \Omega_{E}} f(\omega) \phi_{2}(\omega)
$$

for all increasing functions $f: \Omega_{E} \rightarrow \mathbb{R}$. There are two partial orders of interest on the class of random-cluster measures on $\Omega_{E}$. First,

$$
\phi_{H, \mathbf{p}^{\prime}, q^{\prime}} \leq \phi_{H, \mathbf{p}, q} \text { if } q^{\prime} \geq q, q^{\prime} \geq 1, p_{e}^{\prime} \leq p_{e} \text { for all } e \in E,
$$

and secondly,

$$
\phi_{H, \mathbf{p}^{\prime}, q^{\prime}} \geq \phi_{H, \mathbf{p}, q} \quad \text { if } q^{\prime} \geq q, q^{\prime} \geq 1, \frac{p_{e}^{\prime}}{q^{\prime|e|-1}\left(1-p_{e}^{\prime}\right)} \geq \frac{p_{e}}{q^{|e|-1}\left(1-p_{e}\right)} \text { for all } e \in E
$$

The proofs of these inequalities are just as in Aizenman et al. (1988, Thm 4.1), and they make use (respectively) of the facts that

(i) $k(\omega)$ is a decreasing function of $\omega$ (i.e., $-k(\omega)$ is increasing),

(ii) $k(\omega)+\sum_{e \in E}(|e|-1) \omega(e)$ is an increasing function of $\omega(c f .(2.8))$.

Here, $|e|$ is the cardinality of the set $e$, i.e., the number of vertices in the hyperedge $e$.

(d) Phase transition. It is premature to discuss phase transition at this point, since $H$ is assumed finite at this stage. In the next section, we shall construct a family $\left(\phi_{\Lambda, \mathbf{p}, q}\right)$ of random-cluster measures indexed by certain finite subsets $\Lambda$ of $\mathbb{Z}^{d}$, and we shall take the limit as $\Lambda \uparrow \mathbb{Z}^{d}$. The weak limit of the associated sequence of measures exists if $q \geq 1$, and the question arises as to whether or not the limit measure exhibits a phase transition, for any fixed value of $q$ and as the parameter $\mathbf{p}$ varies. The above comparison inequalities imply the existence of phase transitions for all values of $q(\geq 1)$ whenever it is known that phase transition occurs for some value of $q(\geq 1)$. See Aizenman et al. (1988, Thm 4.2).

\section{Strict inequality for critical values}

Let $d \geq 2$, and let $\mathbb{Z}^{d}$ be the set of $d$-vectors $x=\left(x_{1}, x_{2}, \ldots, x_{d}\right)$ of integers. We begin this section by constructing a family $\left(\phi_{\Lambda, \mathbf{p}, q}\right)$ of random-cluster measures indexed by finite boxes $\Lambda$ contained in $\mathbb{Z}^{d}$; then we shall take the limit as $\Lambda \uparrow \mathbb{Z}^{d}$, and shall consider the phase transition exhibited by the (weak) limit measure

$$
\phi_{\mathbf{p}, q}=\lim _{\Lambda \uparrow \mathbb{Z}^{d}} \phi_{\Lambda, \mathbf{p}, q}
$$


Let $K$ be a finite family of distinct subsets of $\mathbb{Z}^{d}$ satisfying $|e| \geq 2$ for all $e \in K$. Sets in $K$ are the basic 'interaction-sets' of the process; if $|e|=2$ for all $e \in K$, then only pairinteractions occur. The class $K$ generates a family of 'interaction-sets' in $\mathbb{Z}^{d}$, obtained by applying periodic families of translates to the members of $K$. Let $\tau=(\tau(e): e \in K)$ be a sequence of points in $\mathbb{Z}^{d}$, and we assume that each $\tau(e)=\left(\tau_{1}(e), \tau_{2}(e), \ldots, \tau_{d}(e)\right)$ satisfies

$$
\tau_{i}(e)>0 \text { for } 1 \leq i \leq d,
$$

for all $e \in K$. To each $e \in K$, there corresponds a family

$$
T(e)=\left\{e+z \cdot \tau(e): z \in \mathbb{Z}^{d}\right\}
$$

of copies of $e$, obtained by translating $e$ by all (vector) multiples of $\tau(e)$; here and later,

$$
u . v=\left(u_{1} v_{1}, u_{2} v_{2}, \ldots, u_{d} v_{d}\right) \quad \text { for } u, v \in \mathbb{Z}^{d} .
$$

In the simplest situation, we may take $\tau(e)=(1,1, \ldots, 1)$ for all $e$, but many cases of practical interest are more complicated than this. We write $\mathbb{F}=K \times \mathbb{Z}^{d}$. The set $\mathbb{F}$ generates a family of subsets of $\mathbb{Z}^{d}$ given by the mapping $(e, z) \mapsto e+z \cdot \tau(e)$, for $(e, z) \in \mathbb{F}$. This family may contain multiple entries, since there may exist $\left(e_{1}, z_{1}\right),\left(e_{2}, z_{2}\right) \in \mathbb{F}$ such that $e_{1}+z_{1} \cdot \tau\left(e_{1}\right)=e_{2}+z_{2} \cdot \tau\left(e_{2}\right)$. We define $\mathbb{H}$ to be the hypergraph with vertex set $\mathbb{Z}^{d}$ and hyperedge set $(e+z \cdot \tau(e):(e, z) \in \mathbb{F})$, each hyperedge occurring the correct number of times. Upon the hypergraph $\mathbb{H}$ we shall construct the random-cluster measures. For $u, v \in \mathbb{Z}^{d}$, we write $u \sim v$ if there exists $(e, z) \in \mathbb{F}$ with $\{u, v\} \subseteq e+z \cdot \tau(e)$. We say that 'the vertex $w$ is 2-connected to $\infty$ ' if there exist two infinite vertex-disjoint paths, neither containing $w$, but such that their endpoints $u$ and $v$ satisfy $w \sim u$ and $w \sim v$. We make the following assumptions about the family $K$ :

$$
\begin{aligned}
& \text { the infinite hypergraph } \mathbb{H} \text { is connected, } \\
& \text { the origin is } 2 \text {-connected to } \infty \text {. }
\end{aligned}
$$

Assumption (3.2) is vital for what follows. Assumption (3.3) is a matter of convenience only, as the following sketch argument indicates. The hypergraph $\mathbb{H}$ is invariant under translations of the form $x \mapsto x+z \cdot \tau$ for any given $z \in \mathbb{Z}^{d}$, where $\tau=\left(\tau_{1}, \tau_{2}, \ldots, \tau_{d}\right)$ is given by $\tau_{i}=\prod_{e \in K} \tau_{i}(e)$. Therefore, if there exists a vertex which is 2-connected to $\infty$, then there exists a periodic class of such vertices; in this case, we may as well assume that the origin lies in this class. In order to show that such vertices exist, we may argue as follows. We define a doubly-infinite path of $\mathbb{H}$ to be a doubly-infinite sequence $\ldots, v_{-1}, e_{-1}, v_{0}, e_{0}, v_{1}, e_{1}, \ldots$ of distinct vertices $v_{i}$ and distinct hyperedges $e_{j}$ such that $\left\{v_{i}, v_{i+1}\right\} \subseteq e_{i}$ for all $i$. The graph $\mathbb{Z}^{d}$ (considered as a graph with the usual neighbourrelation) contains many doubly-infinite paths, including for example the first coordinate axis. Consequently (using the argument in the proof of the forthcoming Lemma 9) $\mathbb{H}$ must contain a doubly-infinite path lying close to this coordinate axis: more specifically, there exists a constant $D$ and a doubly-infinite path $\pi$ of $\mathbb{H}$ such that each vertex of $\pi$ lies in the tube $\mathbb{Z} \times[-D, D]^{d-1}$. However, every vertex of a doubly-infinite path of $\mathbb{H}$ is 2 -connected to $\infty$.

For a reason which will become evident soon, it is convenient to describe the hypergraph $\mathbb{H}$ in an equivalent but different way than that used above. Let

$$
\kappa_{i}=\operatorname{lcm}\left\{\tau_{i}(e): e \in K\right\}, \quad 1 \leq i \leq d,
$$


the least common multiple of the $\tau_{i}(e)$; we write $\kappa=\left(\kappa_{1}, \kappa_{2}, \ldots, \kappa_{d}\right)$. The hypergraph $\mathbb{H}$ is invariant under translations of $\mathbb{Z}^{d}$ of the form $x \mapsto x+z . \kappa$ for any given $z \in \mathbb{Z}^{d}$. Let

$$
L=\left(e+z \cdot \tau(e): 0 \leq z_{i}<\kappa_{i} / \tau_{i}(e) \text { for } 1 \leq i \leq d, e \in K\right),
$$

a family of translates of the members of $K$; note that the entries of $L$ may be repeated, since there may exist $e, f \in K$ with $e \neq f$ such that $e+z(e) \cdot \tau(e)=f+z(f) \cdot \tau(f)$ for some $z(e), z(f) \in \mathbb{Z}^{d}$ satisfying $0 \leq z_{i}(g)<\kappa_{i} / \tau_{i}(g)$ for $g=e, f$ and $1 \leq i \leq d$. Instead of working with the set $\mathbb{F}=K \times \mathbb{Z}^{d}$, we propose to work with the set $\mathbb{E}=L \times \mathbb{Z}^{d}$; this will aid the calculations to follow. There is a one-one correspondence between $\mathbb{E}$ and $\mathbb{F}$, but a little care is needed to see this, since $L$ may have some entries which are equal. Each pair $(e, z) \in \mathbb{F}$ may be mapped onto a triple $(e, u, v)$ where $u, v\left(\in \mathbb{Z}^{d}\right)$ are given by the factorization

$$
z \cdot \tau(e)=v \cdot \kappa+u \cdot \tau(e), \quad 0 \leq u_{i}<\kappa_{i} / \tau_{i}(e) \text { for } 1 \leq i \leq d .
$$

Since the pair $(u, v)$ specifies $z$ uniquely for any given $e$, we have that $\mathbb{F}$ is in one-one correspondence with the set $I \times \mathbb{Z}^{d}$, where

$$
I=\prod_{e \in K} \prod_{i=1}^{d}\left\{0,1, \ldots,\left(\kappa_{i} / \tau_{i}(e)\right)-1\right\}
$$

is the index set of $L$. Therefore $\mathbb{E}$ and $\mathbb{F}$ are in one-one correspondence; the useful way of thinking about this correspondence is via the mapping

$$
(e, z) \mapsto(e+u \cdot \tau(e), v)
$$

for $(e, z) \in \mathbb{F}$, where $u$ and $v$ are given as above. The set $\mathbb{E}$ generates the same family of hyperedges as before, the pair $(e, z)\left(\in \mathbb{E}=L \times \mathbb{Z}^{d}\right)$ giving rise to the subset $e+z . \kappa$ of $\mathbb{Z}^{d}$. As before, this collection of subsets may contain multiple entries. We emphasize the role of $\mathbb{E}$ by writing $\mathbb{H}$ as the pair $\mathbb{H}=\left(\mathbb{Z}^{d}, \mathbb{E}\right)$; this involves a (slight) abuse of notation, since $\mathbb{E}$ is not itself a set of subsets of $\mathbb{Z}^{d}$. When we speak of a hyperedge $(e, z) \in \mathbb{E}$, we will be referring to the corresponding set $e+z . \kappa$ of vertices.

A configuration $\omega=(\omega(e, z):(e, z) \in \mathbb{E}) \in\{0,1\}^{\mathbb{E}}$ is an allocation of 0 or 1 to each hyperedge of $\mathbb{H}$, and $\Omega$ denotes the set of all such configurations. With $\Omega$ we associate the $\sigma$-field $\mathcal{F}$ generated by the finite-dimensional cylinders. For $\omega \in \Omega$, we write $\eta(\omega)=$ $\{(e, z) \in \mathbb{E}: \omega(e, z)=1\}$, the set of 'open' hyperedges. We shall sometimes confuse the roles of $\omega$ and $\eta(\omega)$, and may speak of ' $\omega$ containing the hyperedge $(e, z)$ ' when we mean that $(e, z) \in \eta(\omega)$. For subsets $A$ and $B$ of $\mathbb{Z}^{d}$, and configurations $\omega$, we write $A \leftrightarrow B$ if there is a path of $\left(\mathbb{Z}^{d}, \eta(\omega)\right)$ joining some point in $A$ to some point in $B$.

Let $\Lambda$ be a finite box of $\mathbb{Z}^{d}$, i.e.,

$$
\Lambda=\prod_{i=1}^{d}\left[x_{i}, y_{i}\right], \quad \text { for some } x, y \in \mathbb{Z}^{d} .
$$

The box $\Lambda$ gives rise to a sub-hypergraph of $\mathbb{H}$ defined as follows. Let $\triangle \Lambda$ be the set of all vertices $v(\notin \Lambda)$ with the property that every infinite path of $\mathbb{H}$ beginning at $v$ contains 
some vertex of $\Lambda$; let $\bar{\Lambda}=\Lambda \cup \triangle \Lambda$. With the set $\bar{\Lambda}$ we associate the set $\mathbb{E}_{\Lambda}$ of all hyperedges $(e, z)(\in \mathbb{E})$ with $e+z . \kappa \subseteq \bar{\Lambda}$. Thus, $\left(\bar{\Lambda}, \mathbb{E}_{\Lambda}\right)$ is the finite sub-hypergraph of $\mathbb{H}$ obtained by adding to $\Lambda$ all vertices which lie in no infinite path of $\mathbb{Z}^{d} \backslash \Lambda$. It turns out to be more convenient to work with the hypergraph $\left(\bar{\Lambda}, \mathbb{E}_{\Lambda}\right)$ rather than with the smaller hypergraph induced by the vertex set $\Lambda$, although in principle the arguments which follow might be reworked along the latter route. We shall see in Lemma 9 that there exists a constant $D$ such that

$$
\Lambda \subseteq \bar{\Lambda} \subseteq \Lambda+[-D, D]^{d}
$$

for all finite boxes $\Lambda$.

We define the boundary $\partial \bar{\Lambda}$ as the set of points $v(\in \bar{\Lambda})$ for which there exists $w(\notin \bar{\Lambda})$ satisfying $v \sim w$; i.e.,

$$
\partial \bar{\Lambda}=\bar{\Lambda} \cap\left\{\bigcup_{(e, z) \in \mathbb{E} \backslash \mathbb{E}_{\Lambda}}(e+z . \kappa)\right\} .
$$

Note that, by the definition of $\bar{\Lambda}$, it is the case that $\partial \bar{\Lambda} \subseteq \Lambda$. The main advantage of working with $\left(\bar{\Lambda}, \mathbb{E}_{\Lambda}\right)$ is that, for all $u_{1}, u_{2} \in \partial \bar{\Lambda}$, there exists a path joining $u_{1}$ and $u_{2}$ using only hyperedges lying in $\mathbb{E} \backslash \mathbb{E}_{\Lambda}$; see Lemma 10 .

Finally, let $\Omega_{\Lambda}$ be the subset of $\Omega$ containing all configurations $\omega$ satisfying $\omega(e, z)=1$ if $(e, z) \notin \mathbb{E}_{\Lambda}$, and write $\mathcal{F}_{\Lambda}$ for the $\sigma$-field of all subsets of $\Omega_{\Lambda}$.

Next we define the random-cluster measures. Let $\mathbf{p}=\left(p_{e}: e \in L\right)$ be a real vector satisfying $0 \leq p_{e} \leq 1$ for all $e \in L$, and let $q$ be a positive real number. The parameters $\mathbf{p}, q$ specify a random-cluster process in the following way. For any finite box $\Lambda$, we let $\phi_{\Lambda, \mathbf{p}, q}$ be the probability measure on $\left(\Omega_{\Lambda}, \mathcal{F}_{\Lambda}\right)$ given by

$$
\phi_{\Lambda, \mathbf{p}, q}(\omega)=\frac{1}{Z_{\Lambda}}\left\{\prod_{(e, z) \in \mathbb{E}_{\Lambda}} p_{e}^{\omega(e)}\left(1-p_{e}\right)^{1-\omega(e)}\right\} q^{k(\omega)}, \quad \omega \in \Omega_{\Lambda},
$$

where $k(\omega)$ is the number of components of the graph $\left(\mathbb{Z}^{d}, \eta(\omega)\right)$, and

$$
Z_{\Lambda}=\sum_{\omega \in \Omega_{\Lambda}}\left\{\prod_{(e, z) \in \mathbb{E}_{\Lambda}} p_{e}^{\omega(e)}\left(1-p_{e}\right)^{1-\omega(e)}\right\} q^{k(\omega)}
$$

is the appropriate normalizing constant. Note that the measure $\phi_{\Lambda, \mathbf{p}, q}$ contains a 'product measure' term together with a 'Radon-Nikodym derivative' $q^{k(\omega)} / Z_{\Lambda}$. It is interesting to note that much of the contents of this paper may be generalized to situations in which this 'derivative' is replaced by one of a large family of non-constant positive functions (see the discussion after Proposition 5). The measure defined in (3.5) is essentially the same as that given in (2.3) and defined on the hypergraph obtained from $\mathbb{H}$ by identifying all vertices not belonging to $\bar{\Lambda} \backslash \partial \bar{\Lambda}$ (see Lemma 10).

We assume henceforth that $q \geq 1$, and we shall occasionally suppress explicit reference to $q$. It may be seen, as in Aizenman et al. (1988), that the limit measure

$$
\phi_{\mathbf{p}, q}=\lim _{\Lambda \uparrow \mathbb{Z}^{d}} \phi_{\Lambda, \mathbf{p}, q}
$$

exists in the weak sense, where the limit is understood to be taken along any increasing sequence of finite boxes $\Lambda$. For any such box $\Lambda$, let

$$
\theta_{\Lambda}(\mathbf{p}, q)=\phi_{\Lambda, \mathbf{p}, q}(0 \leftrightarrow \infty)
$$


where $\{0 \leftrightarrow \infty\}$ is the event that the origin (denoted by 0 ) is in an infinite component. It is the case that

$$
\theta_{\Lambda}(\mathbf{p}, q) \downarrow \theta(\mathbf{p}, q) \quad \text { as } \Lambda \uparrow \mathbb{Z}^{d}
$$

where

$$
\theta(\mathbf{p}, q)=\phi_{\mathbf{p}, q}(0 \leftrightarrow \infty) .
$$

As remarked at the end of the last section, $\phi_{\Lambda, \mathbf{p}, q}$ and $\phi_{\mathbf{p}, q}$ satisfy the FKG inequality, and it follows as in Aizenman et al. (1988) that the quantities $\theta_{\Lambda}(\mathbf{p}, q)$ and $\theta(\mathbf{p}, q)$ are non-decreasing functions of $\mathbf{p}$ when $q \geq 1$.

For $\mathbf{p} \in[0,1]^{L}$ let

$$
\gamma_{c}(\mathbf{p})=\sup \{\gamma \geq 0: \theta(\gamma \mathbf{p}, q)=0\}
$$

The set $\mathrm{C}_{q}=\left\{\mathbf{p} \in[0,1]^{L}: \gamma_{c}(\mathbf{p})=1\right\}$ is called the critical surface of the process, and the sets

$$
\begin{aligned}
& \mathrm{SB}_{q}=\left\{\mathbf{p} \in[0,1]^{L}: \gamma_{c}(\mathbf{p})>1\right\} \\
& \mathrm{SP}_{q}=\left\{\mathbf{p} \in[0,1]^{L}: \gamma_{c}(\mathbf{p})<1\right\}
\end{aligned}
$$

are called the subcritical and supercritical regions. We shall prove a result concerning the behaviour of $\theta(\cdot, q)$ in the neighbourhood of the critical surface. Our result amounts to a characterization of those hyperedges $(e, z) \in \mathbb{E}$ which contribute in a vital way to the phase transition. To this end we introduce a classification of hyperedges in $\mathbb{E}$. For $(e, z) \in \mathbb{E}$, we call the hyperedge $(e, z)$ essential if there exist $u, v \in e$ and two infinite vertex-disjoint paths of $\mathbb{H}$ with endpoints $u+z . \kappa$ and $v+z . \kappa$ (lying in $e+z . \kappa)$. Note that, unlike the case when $|e|=2$ for all $(e, z) \in \mathbb{E}$, the hyperedges of the above paths need not (and generally will not) be distinct. If $(e, z)$ is not essential, we call it inessential. Since $\mathbb{H}$ is invariant under translations of the form $x \mapsto x+z . \kappa$ for given $z \in \mathbb{Z}^{d}$, it is the case that $(e, z)$ is essential if and only if $(e, 0)$ is essential. We denote by $\mathcal{E}$ the set of all $e \in L$ for which $(e, 0)$ is essential, and by $\mathcal{I}$ the set of all $e \in L$ for which $(e, 0)$ is inessential. We write

$$
\overline{\mathcal{E}}=\mathcal{E}+\mathbb{Z}^{d} . \kappa, \quad \overline{\mathcal{I}}=\mathcal{I}+\mathbb{Z}^{d} . \kappa,
$$

the families of all (respectively) essential and inessential hyperedges of $\mathbb{H}$.

Let $\Lambda(n)=[-n, n]^{d}$, and let $\theta_{n}(\mathbf{p}, q)=\theta_{\Lambda(n)}(\mathbf{p}, q)$. For $\mathbf{p} \in \mathbb{R}^{L}$ we define $\widehat{\mathbf{p}}=\left(\widehat{p}_{e}: e \in\right.$ $L)$ by

$$
\widehat{p}_{e}= \begin{cases}p_{e} & \text { if } e \in \mathcal{E} \\ 0 & \text { if } e \in \mathcal{I}\end{cases}
$$

For a subset $F$ of $\mathbb{R}^{L}$, we write $\widehat{F}$ for the set of vectors $\mathbf{q}=\left(q_{e}: e \in \mathcal{E}\right) \in \mathbb{R}^{\mathcal{E}}$ with the property that there exists $\mathbf{p} \in F$ such that $q_{e}=p_{e}$ for all $e \in \mathcal{E}$; thus $\widehat{F}$ is the projection of $F$ with respect to those components indexed by $\mathcal{E}$. Let $U$ be the unit sphere of $\mathbb{R}^{\mathcal{E}}$, with the topology induced from the euclidean topology on $\mathbb{R}^{\mathcal{E}}$. We call a subset $F$ of $\mathbb{R}^{L}$ full if $\widehat{F}$ is an open subset of $U$ which contains the set of all points of $U$ having non-negative coordinates (i.e., all points of $U$ lying in the first orthant of $\mathbb{R}^{\mathcal{E}}$ ). Our main result is the following. 


\section{Theorem 1.}

(a) Let $q \geq 1$. For $\mathbf{p} \in(0,1)^{L}$,

$$
\theta(\mathbf{p}, q)=\theta(\widehat{\mathbf{p}}, q)
$$

(b) Let $q>1$ and let $\mathbf{p} \in \mathrm{C}_{q}$ where $0<p_{e}<1$ for all $e \in \mathcal{E}$. There exist positive constants $\delta, \nu$, and $\epsilon_{0}$, together with a full subset $F$ of $\mathbb{R}^{L}$, such that

$$
\theta(\mathbf{p}+\nu \epsilon \mathbf{g}, q) \leq \theta(\mathbf{p}+\epsilon \mathbf{h}, q) \leq \theta(\mathbf{p}+\delta \epsilon \mathbf{g}, q)
$$

for all $0<\epsilon<\epsilon_{0}$ and all $\mathbf{g}, \mathbf{h} \in F$.

It is a consequence of part (a) that the presence or absence of inessential hyperedges is irrelevant for the existence of infinite paths from the origin. Consequently, in studying the long-range properties of the process with parameter $\mathbf{p}$, it suffices to work with the reduced vector $\widehat{\mathbf{p}}$. Note that, in part (b), the quantities $\delta, \nu, \epsilon_{0}$, and $F$ may depend on the value of $\widehat{\mathbf{p}}$. It is easily seen from part (a) and the definition of $\mathrm{C}_{q}$ that $\theta(\mathbf{p}+\epsilon \mathbf{h}, q)>0$ when $\mathbf{p} \in \mathrm{C}_{q}, \epsilon>0$, and $\mathbf{h}$ is such that $h_{e}>0$ for all $e \in \mathcal{E}$. A consequence of Theorem 1 is therefore the existence, for each $\mathbf{p} \in \mathrm{C}_{q}$ with $p_{e}>0$ for $e \in \mathcal{E}$, of a positive real $\epsilon_{1}$ and a full subset $F$ of $\mathbb{R}^{L}$ such that $(\mathbf{p}+\epsilon F) \cap[0,1]^{L} \subseteq \mathrm{SP}_{q}$ for $0<\epsilon<\epsilon_{1}$.

Part (b) is the principal part of Theorem 1. As remarked in the introduction, the arguments leading to (3.9) fail in the case $q=1$, i.e., in the case of 'many-body' bond percolation. Nevertheless, the arguments of Aizenman and Grimmett (1991) may be adapted in order to derive a corresponding result (via a corresponding version of Theorem 2). There is however an important variation in the case of percolation, in that the collection $\mathcal{E}$ of essential hyperedges must be replaced by a collection $\mathcal{E}^{\prime}$ defined in the following way. For $\omega \in \Omega$ and $e \in L$, let $\omega_{e}, \omega^{e} \in \Omega$ be given by (2.9). We say that $\omega(\in \Omega)$ contains a doubly-infinite path if there exists a doubly-infinite alternating sequence $\ldots, v_{-1}, e_{-1}, v_{0}, e_{0}, v_{1}, e_{1}, \ldots$ of distinct vertices $v_{i}$ and distinct hyperedges $e_{j}$ such that $\left\{v_{i}, v_{i+1}\right\} \subseteq e_{i}$ and $\omega\left(e_{i}\right)=1$ for all $i$. Now, we define $\mathcal{E}^{\prime}$ to be the set of all $e(\in L)$ with the property that there exists $\omega \in \Omega$ such that $\omega^{e}$ contains a doubly-infinite path but $\omega_{e}$ contains no such path. It is clear that $\mathcal{E}^{\prime} \subseteq \mathcal{E}$, and it is sometimes the case that $\mathcal{E}^{\prime} \neq \mathcal{E}$ (for example, in Example 1 at the end of Section 1, we have that $\mathcal{E}=\left\{e_{1}, e_{2}\right\}$ but $\left.\mathcal{E}^{\prime}=\left\{e_{1}\right\}\right)$. The set $\mathcal{E}^{\prime}$ assumes the role of $\mathcal{E}$ in the case $q=1$, and the reason for this is roughly as follows. The hyperedges $(e, z)$ which are relevant for the percolation phase transition are those which have the (geometrical) capacity to be pivotal for the event $\{0 \leftrightarrow \infty\}$, and it may be seen that $(e, z)$ is of this type if and only if $e \in \mathcal{E}^{\prime}$. On the other hand, if $q>1$, a hyperedge can in certain circumstances be relevant for the random-cluster phase transition even though it can never (on geometrical grounds) be pivotal. This occurs if the hyperedge, when present in a configuration, can influence the presence or absence of other hyperedges having the capacity to be pivotal. That is to say, such a hyperedge is relevant by virtue of the (non-constant) term $q^{k(\omega)}$ in the random-cluster measure (3.5), rather than by virtue of its capacity for pivotality.

The principal step in the proof of part (b) of Theorem 1 is the following calculation. 
Theorem 2. Let $q>1$. There exists a continuous function $\alpha:(0,1)^{L} \rightarrow(0, \infty)$ and a positive integer $N$ such that

$$
\frac{\partial \theta_{n}}{\partial p_{e}} \leq \alpha(\mathbf{p}) \frac{\partial \theta_{n}}{\partial p_{f}}
$$

for all $\mathbf{p} \in(0,1)^{L}$, all $n \geq N$, and all pairs $e, f \in \mathcal{E}$.

This theorem resembles closely Theorem 1 of [BGK]. Its proof however is considerably more complicated, owing to its extra generality. In understanding the central role of the family $\mathcal{E}$, various difficulties are encountered. The resolutions of these difficulties seem to require substantial technical arguments involving the relationship between the randomcluster measure and the structure of the hypergraph $\mathbb{H}$.

Before stating the next theorem, we introduce some notation. Given two vectors $\mathbf{x}=$ $\left(x_{i}\right)$ and $\mathbf{y}=\left(y_{i}\right)$, we write $\mathbf{x} \leq \mathbf{y}$ if $x_{i} \leq y_{i}$ for all $i$, and we write $\mathbf{x}<\mathbf{y}$ if $\mathbf{x} \leq \mathbf{y}$ but $\mathbf{x} \neq \mathbf{y}$. A function $\mathbf{p}: \mathbb{R}^{m} \rightarrow \mathbb{R}^{n}$ is called increasing if $\mathbf{p}(\mathbf{x}) \leq \mathbf{p}(\mathbf{y})$ whenever $\mathbf{x} \leq \mathbf{y}$. We say that $\mathbf{p}(\mathbf{x})$ is a strictly increasing function of $x_{i}$ if $\mathbf{p}(\mathbf{x})<\mathbf{p}(\mathbf{y})$ whenever $x_{i}<y_{i}$ and $x_{j} \leq y_{j}$ for all $j \neq i$.

Theorem 1 has a valuable consequence obtained by narrowing the field of application to a one-parameter family of processes. Let $\mathbf{J}=\left(J_{e}: e \in L\right) \in[0, \infty)^{L}$ and let $\beta>0$; we think of $\mathbf{J}$ as describing the strengths of the many-body interactions, and $\beta$ as being the parameter (or 'inverse temperature') of the process. Suppose that $\mathbf{p}$ is a continuous increasing function of the pair $(\mathbf{J}, \beta)$, say $\mathbf{p}=\mathbf{p}(\mathbf{J}, \beta)$. The critical value of $\beta$ is given by

$$
\beta_{c}(\mathbf{J}, q)=\sup \{\beta: \theta(\mathbf{p}(\mathbf{J}, \beta), q)=0\} .
$$

An example of particular importance is the case when

$$
p_{e}=1-e^{-\beta J_{e}}, \quad \text { for } e \in L ;
$$

cf. (2.5). We write $\widehat{\mathbf{p}}$ for the function obtained according to (3.7); i.e., if $\mathbf{p}(\mathbf{J}, \beta)=$ $\left(p_{e}(\mathbf{J}, \beta): e \in L\right)$, then $\widehat{\mathbf{p}}(\mathbf{J}, \beta)=\left(\widehat{p}_{e}(\mathbf{J}, \beta): e \in L\right)$ where

$$
\widehat{p}_{e}(\mathbf{J}, \beta)= \begin{cases}p_{e}(\mathbf{J}, \beta) & \text { if } e \in \mathcal{E} \\ 0 & \text { if } e \in \mathcal{I} .\end{cases}
$$

Theorem 3. Let $q>1$, and suppose that the function $\mathbf{p}:[0, \infty)^{L} \times(0, \infty) \rightarrow[0,1]^{L}$ is continuous and increasing.

(a) It is the case that $\beta_{c}(\mathbf{J}, q)=\beta_{c}\left(\mathbf{J}^{\prime}, q\right)$ if $\widehat{\mathbf{p}}(\mathbf{J}, \beta)=\widehat{\mathbf{p}}\left(\mathbf{J}^{\prime}, \beta\right)$ for all $\beta$.

(b) In addition, $\beta_{c}(\mathbf{J}, q)>\beta_{c}\left(\mathbf{J}^{\prime}, q\right)$ if $\widehat{\mathbf{p}}(\mathbf{J}, \beta)<\widehat{\mathbf{p}}\left(\mathbf{J}^{\prime}, \beta\right)$ and $0<\widehat{p}_{e}(\mathbf{J}, \beta)<1$ for all $\beta>0$.

We now sketch the application of Theorem 3 to Potts models. Let $q$ be an integer satisfying $q \geq 2$, and let $\mathbf{J}=\left(J_{e}: e \in L\right)$ be a vector of strictly positive reals. Write $\Sigma=\{1,2, \ldots, q\}^{\mathbb{Z}^{d}}$, and let $\Sigma_{\Lambda}$ be the set of spin-vectors $\sigma(\in \Sigma)$ satisfying $\sigma(y)=1$ if $y \notin \bar{\Lambda} \backslash \partial \bar{\Lambda}$. We consider the hamiltonian

$$
H_{\Lambda}(\sigma)=\sum_{(e, z) \in \mathbb{E}} J_{e}\left(1-\delta_{\sigma}(e, z)\right), \quad \sigma \in \Sigma_{\Lambda}
$$


where

$$
\delta_{\sigma}(e, z)= \begin{cases}1 & \text { if } \sigma(u)=\sigma(v) \text { whenever } u, v \in e+z . \kappa \\ 0 & \text { otherwise. }\end{cases}
$$

We are interested in the probability measure $\pi_{\Lambda, \mathbf{J}, q}$ given by

$$
\pi_{\Lambda, \mathbf{J}, q}(\sigma)=\frac{1}{Y_{\Lambda}} \exp \left(-\beta H_{\Lambda}(\sigma)\right), \quad \sigma \in \Sigma_{\Lambda},
$$

where $\beta>0$, and $Y_{\Lambda}$ is the appropriate normalizing factor. The weak limit

$$
\pi_{\mathbf{J}, q}^{1}=\lim _{\Lambda \uparrow \mathbb{Z}^{d}} \pi_{\Lambda, \mathbf{J}, q}
$$

exists, and is independent of the choice of increasing sequence of boxes $\Lambda$; see Aizenman et al. (1988) for details of the argument, which is valid for many-body interactions as for pair-interactions (the forthcoming Lemma 10 is relevant here). The order parameter is given by

$$
M(\mathbf{J}, \beta, q)=\frac{q}{q-1}\left(\pi_{\mathbf{J}, q}^{1}(\{\sigma: \sigma(0)=1\})-\frac{1}{q}\right)
$$

and the critical point by

$$
\beta_{c}^{\mathrm{P}}(\mathbf{J}, q)=\sup \{\beta: M(\mathbf{J}, \beta, q)=0\} .
$$

We have that $\pi_{\Lambda, \mathbf{J}, q}(\sigma(y)=1)=1$ for all $y \in \partial \bar{\Lambda}$ and $\sigma \in \Sigma_{\Lambda}$. Writing $H=\left(\bar{\Lambda}, \mathbb{E}_{\Lambda}\right)$, the probability $\pi_{\Lambda, \mathbf{J}, q}(\sigma)$ may be represented as the conditional probability $\pi_{H, \mathbf{p}, q}\left(\sigma_{\Lambda} \mid B\right)$ given by (2.4), where $B=\{\sigma(y)=1$ for all $y \in \partial \bar{\Lambda}\}, \sigma_{\Lambda}=\left(\sigma_{\Lambda}(x): x \in \bar{\Lambda}\right)$ is given by $\sigma_{\Lambda}(x)=\sigma(x)$ for $x \in \bar{\Lambda}$, and $\mathbf{p}$ satisfies (3.12). It follows, by applying (2.6) and (2.7) with $u=0$ and $v$ being a composite vertex obtained by identifying all vertices in $\partial \bar{\Lambda}$ (and making surreptitious use of Lemma 10), that

$$
\pi_{\Lambda, \mathbf{J}, q}(\sigma(0)=1)-\frac{1}{q}=\left(1-q^{-1}\right) \theta_{\Lambda}(\mathbf{p}, q),
$$

whence

$$
M(\mathbf{J}, \beta, q)=\theta(\mathbf{p}, q)
$$

Therefore $\beta_{c}^{\mathrm{P}}(\mathbf{J}, q)=\beta_{c}(\mathbf{p}, q)$ where $\mathbf{p}=\mathbf{p}(\mathbf{J}, q)$ satisfies (3.12). Note that $\widehat{\mathbf{p}}(\mathbf{J}, q)$ is strictly increasing in $J_{e}$ for any $e \in \mathcal{E}$. It is a consequence of Theorems 1 and 3 that $\beta_{c}^{\mathrm{P}}(\mathbf{J}, q)=\beta_{c}^{\mathrm{P}}(\widehat{\mathbf{J}}, q)$ and that $\beta_{c}^{\mathrm{P}}(\mathbf{J}, q)$ is strictly decreasing in any $J_{e}$ for which $e \in \mathcal{E}$. As in Aizenman and Grimmett (1991), the derivatives of $\beta_{c}^{\mathrm{P}}(\mathbf{J}, q)$ with respect to the $J_{e}$ $(e \in \mathcal{E})$ may be bounded above and below by continuous and strictly negative functions.

\section{Russo's formula and time-evolutions for random-cluster processes}

As before, $\Lambda$ is a finite box of $\mathbb{Z}^{d}$, and $\mathbb{E}_{\Lambda}$ is the set of hyperedges (of $\mathbb{E}=L \times \mathbb{Z}^{d}$ ) which are subsets of $\bar{\Lambda}$. On $\left(\Omega_{\Lambda}, \mathcal{F}_{\Lambda}\right)$ we construct a probability measure which is slightly more 
general than that defined by (3.5). Let $\widetilde{\mathbf{p}}=\left(p_{e, z}:(e, z) \in \mathbb{E}_{\Lambda}\right)$ be a vector of reals each of which satisfies $0 \leq p_{e, z} \leq 1$, and define the measure $\widetilde{\phi}$ on $\left(\Omega_{\Lambda}, \mathcal{F}_{\Lambda}\right)$ by

$$
\widetilde{\phi}(\omega)=\frac{1}{Z}\left\{\prod_{(e, z) \in \mathbb{E}_{\Lambda}} p_{e, z}^{\omega(e, z)}\left(1-p_{e, z}\right)^{1-\omega(e, z)}\right\} q^{k(\omega)}, \quad \omega \in \Omega_{\Lambda},
$$

where $Z=Z(\Lambda, \widetilde{\mathbf{p}}, q)$ is the appropriate normalizing factor. The measure $\phi_{\Lambda, \mathbf{p}, q}$ of (3.5) is retrieved by setting $p_{e, z}=p_{e}$ for $e \in L$ and $z \in \mathbb{Z}^{d}$. Thus, in (4.1), each hyperedge $(e, z)$ has an associated independent variable $p_{e, z}$.

We write $I_{A}$ for the indicator function of an event $A$; for $(e, z) \in \mathbb{E}_{\Lambda}$, we write $I_{e, z}$ for the indicator function of the event $\{\omega(e, z)=1\}$, i.e., $I_{e, z}(\omega)=\omega(e, z)$ for $\omega \in \Omega$.

Proposition 4. Let $0<q<\infty$, and let $A \in \mathcal{F}_{\Lambda}$. If $0<p_{e, z}<1$, then

$$
\frac{\partial}{\partial p_{e, z}} \widetilde{\phi}(A)=\frac{1}{p_{e, z}\left(1-p_{e, z}\right)} \operatorname{cov}\left(I_{e, z}, I_{A}\right) \quad \text { for all }(e, z) \in \mathbb{E}_{\Lambda} \text {. }
$$

Here, 'cov' denotes covariance relative to the probability measure $\widetilde{\phi}$. In the case $q=1$, equation (4.2) is a version of Russo's formula for percolation, but in a form first discovered by Barlow and Proschan (1965, p. 10); see Grimmett (1989, p. 38). It is notable that (4.2) is valid for all values of $q$. Indeed, (4.2) is valid for any measure of the form of (4.1) with the term $q^{k(\omega)}$ replaced by an arbitrary function of $\omega$ which does not depend on $p_{e, z}$ (see also the discussion after Proposition 5). We omit the proof of the above proposition, which may be taken directly from [BGK]. The assumption that $0<p_{e, z}<1$ may be relaxed; see the final paragraph of this section. The bulk of the remainder of this section is devoted to a representation for the covariance in (4.2), when $A$ is an increasing event; this representation is essentially the same as appears in Section 3 of [BGK], but differs in the important detail of condition (4.13).

Suppose that $q \geq 1$ and let $A$ be an increasing event of $\left(\Omega_{\Lambda}, \mathcal{F}_{\Lambda}\right)$ (that is, if $\omega \in A$ and $\omega \leq \omega^{\prime}$ then $\left.\omega^{\prime} \in A\right)$. It will be useful to have recourse to a representation for $\partial \widetilde{\phi}(A) / \partial p_{e, z}$ containing only non-negative terms. Suppose that $0<p_{e, z}<1$ for all $(e, z) \in \mathbb{E}_{\Lambda}$. Using (4.2), we may write

$$
\frac{\partial}{\partial p_{e, z}} \widetilde{\phi}(A)=\frac{\widetilde{\phi}(A)}{p_{e, z}\left(1-p_{e, z}\right)}\{\widetilde{\phi}(\omega(e, z)=1 \mid A)-\widetilde{\phi}(\omega(e, z)=1)\} .
$$

The term in braces is non-negative (by the FKG inequality) and may be represented in terms of coupled time-evolutions of the random-cluster process. We show next how this may be done.

We shall construct certain Markov chains on the state space $\Omega_{\Lambda}$. Let $\mu$ be a probability measure on $\left(\Omega_{\Lambda}, \mathcal{F}_{\Lambda}\right)$, and let $G: \Omega_{\Lambda} \times \Omega_{\Lambda} \rightarrow[0, \infty)$ be the generator of a Markov chain (i.e., for $\omega \neq \omega^{\prime}, G\left(\omega, \omega^{\prime}\right)$ represents the jump-rate of the chain from $\omega$ to $\omega^{\prime}$, while a diagonal element $G(\omega, \omega)$ is chosen in such a way that $\left.\sum_{\omega^{\prime}} G\left(\omega, \omega^{\prime}\right)=0\right)$. If the pair $(\mu, G)$ satisfies the balance equations

$$
\mu(\omega) G\left(\omega, \omega^{\prime}\right)=\mu\left(\omega^{\prime}\right) G\left(\omega^{\prime}, \omega\right) \text { for all } \omega, \omega^{\prime} \in \Omega_{\Lambda},
$$

then the chain is reversible with stationary measure $\mu$. 
For $\omega \in \Omega_{\Lambda}$ and $(f, y) \in \mathbb{E}_{\Lambda}$, we define the configurations $\omega_{f, y}$ and $\omega^{f, y}$ by

$$
\begin{aligned}
& \omega_{f, y}(g, x)= \begin{cases}\omega(g, x) & \text { if }(g, x) \neq(f, y) \\
0 & \text { if }(g, x)=(f, y),\end{cases} \\
& \omega^{f, y}(g, x)= \begin{cases}\omega(g, x) & \text { if }(g, x) \neq(f, y) \\
1 & \text { if }(g, x)=(f, y) .\end{cases}
\end{aligned}
$$

We define

$$
D_{f, y}(\omega)=k\left(\omega_{f, y}\right)-k\left(\omega^{f, y}\right),
$$

the increment in the number of components of the configuration depending on whether or not it contains the hyperedge $(f, y)(c f .(2.8))$.

There are two instances of (4.4) of importance for (4.3). When $\mu=\widetilde{\phi}$, equations (4.4) are satisfied when we take for $G$ the function $H: \Omega_{\Lambda} \times \Omega_{\Lambda} \rightarrow[0, \infty)$ given by

$$
\begin{aligned}
& H\left(\omega_{f, y}, \omega^{f, y}\right)=1 \\
& H\left(\omega^{f, y}, \omega_{f, y}\right)=\frac{\widetilde{\phi}\left(\omega_{f, y}\right)}{\widetilde{\phi}\left(\omega^{f, y}\right)}=\frac{1-p_{f, y}}{p_{f, y}} q^{D_{f, y}(\omega)}
\end{aligned}
$$

for $\omega \in \Omega_{\Lambda}$ and $(f, y) \in \mathbb{E}_{\Lambda}$, where $H\left(\omega, \omega^{\prime}\right)$ is set to 0 for other pairs $\omega, \omega^{\prime}$ with $\omega \neq \omega^{\prime}$.

In the second instance, we take $\mu=\widetilde{\phi}(\cdot \mid A)$, and in this case (4.4) is satisfied by the generator $H^{A}$ given by

$$
H^{A}\left(\omega, \omega^{\prime}\right)=H\left(\omega, \omega^{\prime}\right) I_{A}\left(\omega \wedge \omega^{\prime}\right) \text { if } \omega \neq \omega^{\prime}
$$

where $\omega \wedge \omega^{\prime}(g, x)=\min \left\{\omega(g, x), \omega^{\prime}(g, x)\right\}$ for $(g, x) \in \mathbb{E}_{\Lambda}$.

These two instances give rise to Markov chains which can be constructed on the same sample space in such a way that the first 'lies beneath' the second. To this end we argue as follows.

Let $\Theta_{\Lambda}$ be the set of all pairs $(\pi, \omega)$ of configurations in $\Omega_{\Lambda}$ satisfying $\pi \leq \omega$. We define $J: \Theta_{\Lambda} \times \Theta_{\Lambda} \rightarrow[0, \infty)$ by

$$
\begin{aligned}
J\left(\pi_{f, y}, \omega ; \pi^{f, y}, \omega^{f, y}\right) & =1, \\
J\left(\pi, \omega^{f, y} ; \pi_{f, y}, \omega_{f, y}\right) & =H^{A}\left(\omega^{f, y}, \omega_{f, y}\right), \\
J\left(\pi^{f, y}, \omega^{f, y} ; \pi_{f, y}, \omega^{f, y}\right) & =H\left(\pi^{f, y}, \pi_{f, y}\right)-H^{A}\left(\omega^{f, y}, \omega_{f, y}\right),
\end{aligned}
$$

for all $(\pi, \omega) \in \Theta_{\Lambda}$ and $(f, y) \in \mathbb{E}_{\Lambda}$; all other off-diagonal values of $J$ are set to 0 . Equation (4.9) specifies that, for $\pi \in \Omega_{\Lambda}$ and $(f, y) \in \mathbb{E}_{\Lambda}$, the hyperedge $(f, y)$ is acquired by $\pi$ (if it does not already contain it) at rate 1; any hyperedge so acquired is added also to $\omega$ if it does not already contain it. Equation (4.10) specifies that, for $\omega \in \Omega_{\Lambda}$ and $(f, y) \in \eta(\omega)$, the hyperedge $(f, y)$ is removed from $\omega$ (and also from $\pi$ if $(f, y) \in \eta(\pi)$ ) at the rate given by (4.10). For $(f, y) \in \eta(\pi)(\subseteq \eta(\omega))$, there is an additional rate at which $(f, y)$ is removed from $\pi$ but not from $\omega$. Note that this additional rate (given in (4.11)) is indeed non-negative, since

$$
H\left(\pi^{f, y}, \pi_{f, y}\right)-H^{A}\left(\omega^{f, y}, \omega_{f, y}\right)=\frac{1-p_{f, y}}{p_{f, y}}\left\{q^{D_{f, y}(\pi)}-q^{D_{f, y}(\omega)} I_{A}\left(\omega_{f, y}\right)\right\} \geq 0,
$$


by (4.7) and (4.8); remember that $q \geq 1$ and $D_{f, y}(\omega) \leq D_{f, y}(\pi)$ when $\pi \leq \omega$. This additional rate is strictly positive if and only if

$$
\text { either } \omega_{f, y} \notin A \text {, or } \omega_{f, y} \in A \text { and } D_{f, y}(\pi)>D_{f, y}(\omega) \text {. }
$$

We note also that the transitions referred to in (4.9)-(4.11) take pairs $(\pi, \omega)$ with $\pi \leq \omega$ onto new pairs $\left(\pi^{\prime}, \omega^{\prime}\right)$ with $\pi^{\prime} \leq \omega^{\prime}$. Therefore, the function $J$ is the generator of a Markov chain $\left(X_{t}, Y_{t}\right)_{t \geq 0}$ taking values in $\Theta_{\Lambda}$. We assume henceforth that $\left(X_{t}, Y_{t}\right)_{t \geq 0}$ is such a chain, and we set $\left(X_{0}, Y_{0}\right)=(\mathbf{0}, \mathbf{1})$ where $\mathbf{i}\left(\in \Omega_{\Lambda}\right)$ is the configuration which takes the value $i$ on every hyperedge in $\mathbb{E}_{\Lambda}$. We write $P$ for the appropriate probability measure on the set of sample paths of this process.

By further examination of (4.9)-(4.11), it is easily seen that $X=\left(X_{t}\right)_{t \geq 0}$ is a Markov chain on $\Omega_{\Lambda}$ with generator $H$, and stationary measure $\widetilde{\phi}$. Similarly, $Y=\left(Y_{t}\right)_{t \geq 0}$ is a Markov chain on the subset $A$ of $\Omega_{\Lambda}$ with generator given by $H^{A}$ restricted to $A \times A$, and stationary measure $\widetilde{\phi}(\cdot \mid A)$. Both these chains are irreducible. This claim is trivial for $X$, since each hyperedge has a strictly positive birth and death rate in this process. The claim is also true for $Y$, as the following argument indicates. If $\omega \in A$ and $Y_{t}=\omega$, then the process $Y$ can progress (with a strictly positive probability) from $\omega$ to the state $\mathbf{1}$; this is valid because hyperedges are acquired by $Y$ at rate 1 , and furthermore $\mathbf{1} \in A$ by virtue of the facts that $\omega \in A$ and $A$ is an increasing event. If $\omega^{\prime} \in A$, then $Y$ can progress from 1 to $\omega^{\prime}$ by the removal of exactly those hyperedges $(f, y)$ for which $\omega^{\prime}(f, y)=0$; such transitions have strictly positive jump-rates since $A$ is an increasing event.

It follows from the irreducibility of $X$ and $Y$, and from the facts that $\widetilde{\phi}$ and $\widetilde{\phi}(\cdot \mid A)$ are stationary measures for $X$ and $Y$ respectively, that

$\lim _{t \rightarrow \infty} P\left(X_{t}(e, z)=1\right)=\widetilde{\phi}(\omega(e, z)=1), \quad$ and $\quad \lim _{t \rightarrow \infty} P\left(Y_{t}(e, z)=1\right)=\widetilde{\phi}(\omega(e, z)=1 \mid A)$,

for $(e, z) \in \mathbb{E}_{\Lambda}(c f$. Doob (1953, Thm VI.1.1)).

Finally we recall that $X_{t} \leq Y_{t}$ for all $t$, in the light of which (4.3) may be written in the form given as follows.

Proposition 5. Suppose that $0<p_{e, z}<1$ for all $(e, z) \in \mathbb{E}_{\Lambda}$. If $A$ is an increasing event and $(e, z) \in \mathbb{E}_{\Lambda}$, then

$$
\begin{aligned}
\frac{\partial}{\partial p_{e, z}} \widetilde{\phi}(A) & =\frac{\widetilde{\phi}(A)}{p_{e, z}\left(1-p_{e, z}\right)} \lim _{t \rightarrow \infty}\left\{P\left(Y_{t}(e, z)=1\right)-P\left(X_{t}(e, z)=1\right)\right\} \\
& =\frac{\widetilde{\phi}(A)}{p_{e, z}\left(1-p_{e, z}\right)} \lim _{t \rightarrow \infty}\left\{P\left(X_{t}(e, z)=0, Y_{t}(e, z)=1\right)\right\},
\end{aligned}
$$

where $\left(X_{t}, Y_{t}\right)_{t \geq 0}$ is a Markov chain on $\Omega_{\Lambda} \times A$ with generator given by (4.9)-(4.11) and satisfying $\left(X_{0}, Y_{0}\right)=(\mathbf{0}, \mathbf{1})$.

It is interesting to note that the main results of this paper are valid in considerable extra generality than the random-cluster processes only. Let us consider a measure $\nu$ on $\left(\Omega_{\Lambda}, \mathcal{F}_{\Lambda}\right)$ given by

$$
\nu(\omega)=\frac{1}{Z^{\prime}}\left\{\prod_{(e, z) \in \mathbb{E}_{\Lambda}} p_{e, z}^{\omega(e, z)}\left(1-p_{e, z}\right)^{1-\omega(e, z)}\right\} \rho(\omega), \quad \omega \in \Omega_{\Lambda},
$$


i.e., (4.1) with $q^{k(\omega)}$ replaced by some function $\rho(\omega)$ which does not depend on $\widetilde{\mathbf{p}}$. It may be shown that Proposition 4 and (by Holley (1974)) the argument leading to Proposition 5 are valid so long as $\rho$ satisfies

$$
\rho\left(\omega \vee \omega^{\prime}\right) \rho\left(\omega \wedge \omega^{\prime}\right) \geq \rho(\omega) \rho\left(\omega^{\prime}\right) \quad \text { for } \omega, \omega^{\prime} \in \Omega_{\Lambda}
$$

Subject to certain extra conditions on $\rho$, the remaining analysis of this paper may be validated for measures of the form (4.15) and their weak limits, leading thereby to generalizations of Theorems 1 and 2 .

There appeared at the end of Section 1 certain remarks concerned with the equality of critical exponents for Potts models, when some parameter $J_{e}$ takes the value 0 . In the associated random-cluster process, the corresponding parameter $p_{e}=1-e^{-\beta J_{e}}$ takes the value $p_{e}=0$. In proving these remarks, one needs a version of Proposition 4 which is valid at the point $p_{e, z}=0$, for a given $(e, z) \in \mathbb{E}_{\Lambda}$. For simplicity of notation, we write $\widetilde{\phi}_{p}$ for the measure $\widetilde{\phi}$ in (4.1) with $p_{e, z}=p$; thus, although $\widetilde{\phi}$ depends on $\widetilde{\mathbf{p}}$ (and therefore on $p$ ), we shall think of it as a function of the real parameter $p$ alone. It is an elementary calculation that (4.2) may be replaced by the formula

$$
\widetilde{\phi}_{p}^{\prime}(A)=\frac{S(B) S\left(B^{c}\right)}{\left(p S(B)+(1-p) S\left(B^{c}\right)\right)^{2}}\left\{\widetilde{\phi}_{1}(A)-\widetilde{\phi}_{0}(A)\right\}, \quad 0 \leq p \leq 1,
$$

where $\widetilde{\phi}_{p}^{\prime}(A)$ is the derivative (with respect to $x$ ) of $\widetilde{\phi}_{x}(A)$, evaluated at $x=p, B$ is the event $\left\{\omega \in \Omega_{\Lambda}: \omega(e, z)=1\right\}$, and

$$
\begin{aligned}
S(C) & =\sum_{\omega \in C} \alpha(\omega), \quad C \subseteq \Omega_{\Lambda}, \\
\alpha(\omega) & =\left\{\prod_{\substack{(f, y) \in \mathbb{E}_{\Lambda} \\
(f, y) \neq(e, z)}} p_{f, y}^{\omega(f, y)}\left(1-p_{f, y}\right)^{1-\omega(f, y)}\right\} q^{k(\omega)}, \quad \omega \in \Omega_{\Lambda} .
\end{aligned}
$$

Note that the normalizing factor in (4.1) is given by

$$
Z(p)=p S(B)+(1-p) S\left(B^{c}\right), \quad 0 \leq p \leq 1,
$$

where we have written $Z=Z(p)$ to emphasize the parameter $p$. Therefore

$$
\widetilde{\phi}_{p}^{\prime}(A)=\left\{\frac{Z\left(\frac{1}{2}\right)}{Z(p)}\right\}^{2} \widetilde{\phi}_{1 / 2}^{\prime}(A), \quad 0 \leq p \leq 1,
$$

a formula which expresses the dependence of $\widetilde{\phi}_{p}^{\prime}(A)$ on $p$. The events $B$ and $B^{c}$ may be put in one-one correspondence by the mapping $\omega^{e, z} \mapsto \omega_{e, z}$ between configurations. It is then immediate, using (2.8), that

$$
1 \leq \frac{S\left(B^{c}\right)}{S(B)} \leq q^{|e|-1} .
$$


Hence

$$
\min \left\{1, \frac{1+q^{|e|-1}}{2\left(p+(1-p) q^{|e|-1}\right)}\right\} \leq \frac{Z\left(\frac{1}{2}\right)}{Z(p)} \leq \max \left\{1, \frac{1+q^{|e|-1}}{2\left(p+(1-p) q^{|e|-1}\right)}\right\}, \quad 0 \leq p \leq 1,
$$

by (4.18). Taken in conjunction with (4.19), this implies in particular that

$$
\frac{1}{4}\left(1+\frac{1}{q^{|e|-1}}\right)^{2} \leq \frac{\widetilde{\phi}_{0}^{\prime}(A)}{\widetilde{\phi}_{1 / 2}^{\prime}(A)} \leq 1
$$

which is to say that $\widetilde{\phi}_{0}^{\prime}(A)$ differs from $\widetilde{\phi}_{1 / 2}^{\prime}(A)$ by a multiplicative factor which is bounded above and beneath by positive functions of $q$ alone. Using this observation, one may adapt the arguments of the next two sections in order to obtain the conclusions described at the end of Section 1.

\section{Preliminary lemmas}

In proving Theorems 1-3, we shall make use of certain preliminary results largely concerned with the graph-theoretic structure of $\mathbb{H}$. Our first lemma concerns random-cluster measures on hypergraphs which contain articulation points. Let $H_{1}=\left(V_{1}, E_{1}\right)$ and $H_{2}=\left(V_{2}, E_{2}\right)$ be hypergraphs with exactly one common vertex, i.e., $V_{1} \cap V_{2}=\{v\}$. Let $\mathbf{p}_{1}=\left(p_{e}: e \in E_{1}\right)$ and $\mathbf{p}_{2}=\left(p_{f}: f \in E_{2}\right)$ be vectors satisfying $0 \leq p_{g} \leq 1$ for all $g \in E_{1} \cup E_{2}$, and let $q>0$. Let $\phi_{i}$ be the random-cluster measure on the set $\Omega_{i}=\{0,1\}^{E_{i}}$ of configurations of $H_{i}$ with parameters $\mathbf{p}_{i}$ and $q$; let $\phi$ be the random-cluster measure on $\Omega_{1} \times \Omega_{2}$, thought of as the set of hyperedge-configurations of the hypergraph $H=\left(V_{1} \cup V_{2}, E_{1} \cup E_{2}\right)$, with parameters $\mathbf{p}=\left(p_{g}: g \in E_{1} \cup E_{2}\right)$ and $q$. See (2.3) for the formal definition of these measures.

Lemma 6. We have that $\phi=\phi_{1} \times \phi_{2}$, which is to say that the random-cluster processes on $H_{1}$ and $H_{2}$ are independent.

Proof. Since $V_{1}$ and $V_{2}$ have a single vertex in common, we have that

$$
k\left(\omega_{1} \times \omega_{2}\right)=k\left(\omega_{1}\right)+k\left(\omega_{2}\right)-1, \quad \text { for } \omega_{i} \in \Omega_{i}, i=1,2,
$$

where $k\left(\omega_{i}\right)$ is the number of components of the hypergraph $\left(V_{i}, \eta\left(\omega_{i}\right)\right), i=1,2$, and $\eta\left(\omega_{i}\right)=\left\{e \in E_{i}: \omega_{i}(e)=1\right\}$. It follows from (2.3) that

$$
\phi\left(\omega_{1} \times \omega_{2}\right)=\phi_{1}\left(\omega_{1}\right) \phi\left(\omega_{2}\right)
$$

as required.

The next five lemmas are graph-theoretic. Remember that $\overline{\mathcal{E}}$ is the set of all essential hyperedges; let

$$
V=\bigcup\{e: e \in \overline{\mathcal{E}}\}
$$

and write $\mathbb{H}_{\mathcal{E}}$ for the hypergraph $(V, \overline{\mathcal{E}})$. We may obtain $\mathbb{H}_{\mathcal{E}}$ by deletion of all inessential hyperedges and all vertices which lie in inessential hyperedges only. Let $(e, z) \in \overline{\mathcal{I}}$, the set of inessential hyperedges. By Menger's theorem and assumptions (3.2)-(3.3), there exists a non-empty set $C(e, z)\left(\subseteq \mathbb{Z}^{d}\right)$ such that every infinite path $\pi$ of $\mathbb{H}$ which intersects the 
subset $e+z . \kappa$ of vertices has the property that its vertex set $V(\pi)$ satisfies $C(e, z) \subseteq V(\pi)$. We choose $C(e, z)$ to be maximal with this property, and we have by translation-invariance that $C(e, z)=C(e, 0)+z \cdot \kappa ; C(e, z)$ is exactly the set of all vertices whose removal from $\mathbb{H}$ leaves each (remaining) member of $e+z . \kappa$ in some finite component of the resulting hypergraph. As a consequence of the definition of $C(e, z)$, the points therein may be placed in an order, $c_{1}, c_{2}, \ldots$, in such a way that, for all $n$, every path joining a vertex of $e+z . \kappa$ to $c_{n+1}$ passes through $c_{n}$ (see Figure 2). Also, $C(e, z)$ is finite since, if $C(e, z)$ were infinite, then, by the connectedness of $\mathbb{H}$, all hyperedges in $\mathbb{E}$ would be inessential, in contradiction of (3.3). We define $c(e, z)$ to be the final vertex in the list $c_{1}, c_{2}, \ldots$ defined above. Let $g(e, z)$ be the set of all endpoints of paths of $\mathbb{H}$ which contain vertices in $e+z . \kappa$ but not the vertex $c(e, z)$, and let $h(e, z)$ be the set of all hyperedges of $\mathbb{H}$ which are subsets of $\bar{g}(e, z)=g(e, z) \cup\{c(e, z)\}$. Note that every hyperedge of $h(e, z)$ is inessential, since every infinite path intersecting $g(e, z)$ passes through $c(e, z)$.

Lemma 7. Let $\pi$ be a finite or infinite path of $\mathbb{H}$.

(a) If $\pi$ is finite and both of its endpoints lie in essential hyperedges, then all of the hyperedges of $\pi$ are essential.

(b) If $\pi$ is infinite, and its single endvertex lies in an essential hyperedge, then all of the hyperedges of $\pi$ are essential.

Proof. (a) Let $\pi$ be a path whose endpoints $u$ and $v$ lie in essential hyperedges, and assume that $\pi$ contains at least one hyperedge. Note that $u \neq v$ by the definition of a path. Suppose that there exists an inessential hyperedge $(e, z)$ in $\pi$. It follows that $u, v \notin g(e, z)$, since vertices of $g(e, z)$ lie in only inessential hyperedges. Therefore, $u, v \in \mathbb{Z}^{d} \backslash g(e, z)$. However, all paths from $g(e, z)$ to the set $\{u, v\}$ pass through the articulation point $c(e, z)$, in contradiction of the existence of $\pi$.

(b) Let $\pi$ be an infinite path whose endpoint $u$ lies in an essential hyperedge. Suppose that there exists an inessential hyperedge $(e, z)$ in $\pi$. By the above argument, we have that $u \in \mathbb{Z}^{d} \backslash g(e, z)$, and as above there can be no infinite path from $u$ which uses two or more vertices of $e+z . \kappa$.

Lemma 8. The hypergraph $\mathbb{H}_{\mathcal{E}}=(V, \overline{\mathcal{E}})$ is connected, and the vertex $z . \kappa$ lies in $V$ for all $z \in \mathbb{Z}^{d}$.

Proof. It is a consequence of (3.3) that the origin lies in some essential hyperedge, and therefore lies in $V$ also. As observed previously, $\mathbb{H}$ is invariant under lattice shifts of the form $x \mapsto x+z . \kappa$ for given $z \in \mathbb{Z}^{d}$. Therefore $\mathbb{H}_{\mathcal{E}}$ is similarly invariant, implying that $z . \kappa \in V$ for all $z \in \mathbb{Z}^{d}$.

Let $u, v \in V$, and let $\pi$ be a path of $\mathbb{H}$ joining $u$ to $v$. Each of the vertices $u, v$ lies in some essential hyperedge, and therefore all hyperedges of $\pi$ are essential by Lemma 7 . It follows that $u$ and $v$ are in the same component of $\mathbb{H}_{\mathcal{E}}$, whence $\mathbb{H}_{\mathcal{E}}$ is connected.

For $x \in \mathbb{Z}^{d}$, let

$$
\|x\|=\max \left\{\left|x_{i}\right|: 1 \leq i \leq d\right\} .
$$

Given two sets $A, B \subseteq \mathbb{Z}^{d}$, we define

$$
d(A, B)=\max \{d(x, B): x \in A\}
$$

where

$$
d(x, B)=\min \{\|x-y\|: y \in B\} .
$$


We recall the notation $\Lambda(n)=[-n, n]^{d}$, and we point out that $d(A, B) \leq D$ if and only if $A \subseteq B+\Lambda(D)$. For a path $\pi$ of a hypergraph $H$, we write $V(\pi)$ and $V_{\mathrm{h}}(\pi)$ for the set of vertices of $\pi$, and the set of all vertices lying in hyperedges of $\pi$, respectively. It is important to remember that $V(\pi)$ is not generally the same as $V_{\mathrm{h}}(\pi)$; however, $V(\pi) \subseteq V_{\mathrm{h}}(\pi)$.

Lemma 9. There exists a positive integer $D$ such that the following holds. For all $u, v \in$ $\mathbb{Z}^{d}$ and any path $\pi$ of $\mathbb{Z}^{d}$ joining $u$ to $v$ (where $\mathbb{Z}^{d}$ is thought of as a graph with the usual nearest-neighbour relation)

(a) there exists a path $\widehat{\pi}$ of $\mathbb{H}$ joining $u$ to $v$ such that $d\left(V(\pi), V_{\mathrm{h}}(\widehat{\pi})\right)<D$, and

(b) if $u, v \in V$, then there exists a path $\pi^{\prime}$ of $\mathbb{H}_{\mathcal{E}}$ joining $u$ to $v$ such that $d\left(V(\pi), V_{\mathrm{h}}\left(\pi^{\prime}\right)\right)<$ $D$.

With this choice of $D$, it is the case that

$$
\Lambda \subseteq \bar{\Lambda} \subseteq \Lambda+\Lambda(D)
$$

for all bounded boxes $\Lambda$ of $\mathbb{Z}^{d}$.

Proof. By virtue of (3.2) and the invariance of $\mathbb{H}$ under translations by multiples of $\kappa$, there exists a constant $D_{1}=D_{1}(K)$ such that any two vertices $u, v \in \mathbb{Z}^{d}$ with $\|u-v\|=1$ are connected in $\mathbb{H}$ by a path $\pi_{u v}$ for which $V_{\mathrm{h}}\left(\pi_{u v}\right) \subseteq\{v\}+\Lambda\left(D_{1}\right)$. It follows that, for any pair $u, v \in \mathbb{Z}^{d}$, and for any path $\pi$ from $u$ to $v$ on the graph $\mathbb{Z}^{d}$ with the usual neighbourrelation, there exists a path $\widehat{\pi}$ of $\mathbb{H}$ joining $u$ to $v$ such that $V_{\mathrm{h}}(\widehat{\pi}) \subseteq V(\pi)+\Lambda\left(D_{1}\right)$. Part (a) follows for any $D$ satisfying $D>D_{1}$. The proof of (b) is identical to that of (a), replacing $\mathbb{H}$ by $\mathbb{H}_{\mathcal{E}}$, and (3.2) by Lemma 8 .

Turning to the final assertion of the lemma, let $\Lambda$ be a finite box, and let $\bar{\Lambda}$ be defined as before (see the text between (3.4) and (3.5)). Suppose that $y \notin \Lambda+\Lambda(D)$. There exists an infinite path $\pi$ of $\mathbb{Z}^{d}$ (with the usual neighbour-relation) with endpoint $y$ and containing no vertex of $\Lambda+\Lambda(D)$. By the proof of (a) above, there exists an infinite path of $\mathbb{H}$ with endpoint $y$ and containing no vertex of $\Lambda$. It follows that $y \notin \Delta \Lambda$, implying that $y \notin \bar{\Lambda}=\Lambda \cup \triangle \Lambda$. Therefore $\bar{\Lambda} \subseteq \Lambda+\Lambda(D)$ as required. in $\bar{\Lambda}$.

Let $\Lambda$ be a finite box of $\mathbb{Z}^{d}$, and let $\mathcal{E}_{\Lambda}=\mathbb{E}_{\Lambda} \cap \overline{\mathcal{E}}$, the set of essential hyperedges contained

\section{Lemma 10.}

(a) If $u_{1}, u_{2} \in \partial \bar{\Lambda}$, then there exists a path of $\mathbb{H}$ joining $u_{1}$ to $u_{2}$ which contains only hyperedges lying in $\mathbb{E} \backslash \mathbb{E}_{\Lambda}$.

(b) If furthermore $u_{1}, u_{2} \in V \cap \partial \bar{\Lambda}$, then there exists a path of $\mathbb{H}_{\mathcal{E}}$ joining $u_{1}$ to $u_{2}$ which contains only hyperedges lying in $\overline{\mathcal{E}} \backslash \mathcal{E}_{\Lambda}$.

Proof. (a) Suppose $u_{1}, u_{2} \in \partial \bar{\Lambda}$; we may assume that $u_{1} \neq u_{2}$. By the definition of $\partial \bar{\Lambda}$, there exist infinite paths $\pi_{1}$ and $\pi_{2}$ of $\mathbb{H}$ with respective endpoints $u_{1}$ and $u_{2}$, and which contain no other vertices of $\partial \bar{\Lambda}$ (and therefore $\pi_{1}$ and $\pi_{2}$ contain no hyperedges of $\mathbb{E}_{\Lambda}$ ). Since $\pi_{i}$ is infinite, it contains some vertex $v_{i}$ lying outside $\Lambda(m+D)$, where $D$ is given in Lemma 9 , and $m=\min \{k: \bar{\Lambda} \subseteq \Lambda(k)\}$. Now, there exists a path of $\mathbb{Z}^{d}$ (as a graph with the usual neighbour-relation) from $v_{1}$ to $v_{2}$ which is disjoint from $\Lambda(m+D)$. By Lemma 9 (a), there exists a path $\pi_{3}$ of $\mathbb{H}$ joining $v_{1}$ to $v_{2}$ which uses no hyperedges of $\mathbb{E}_{\Lambda}$. The union of $\pi_{3}$, together with the portion of each $\pi_{i}$ between $u_{i}$ and $v_{i}$, contains a path of the required type joining $u_{1}$ to $u_{2}$. 
(b) Let $u_{1}, u_{2} \in V \cap \partial \bar{\Lambda}$, and $u_{1} \neq u_{2}$. By virtue of the assumption that $u_{1}, u_{2} \in \partial \bar{\Lambda}$, there exist infinite paths $\pi_{1}$ and $\pi_{2}$ of $\mathbb{H}$ with respective endvertices $u_{1}$ and $u_{2}$, and containing no other vertices of $\partial \bar{\Lambda}$. Since $u_{1}, u_{2} \in V$, Lemma 7 implies that all hyperedges contained in $\pi_{1}$ and $\pi_{2}$ are essential. The proof is now finished as was part (a), this time by applying Lemma $9(\mathrm{~b})$.

Given two subsets $A_{1}$ and $A_{2}$ of $\mathbb{Z}^{d}$, we say that ' $A_{1}$ is 2 -connected to $A_{2}$ ' if there exist two vertex-disjoint paths $\pi_{i}=\left(a_{i, 1}, g_{i, 1}, a_{i, 2}, \ldots, g_{i, r_{i}}, a_{i, r_{i}+1}\right), i=1,2$, where the $a_{i, j}$ are vertices and the $g_{i, j}$ are hyperedges, such that $a_{i, 1} \in A_{1}$ and $a_{i, r_{i}+1} \in A_{2}$ for $i=1,2$. Note that the paths $\pi_{i}$ are not required to be hyperedge-disjoint. If the above holds, we write $A_{1} \longrightarrow A_{2}$. If $\left|A_{1} \cap A_{2}\right| \geq 2$ then it is immediate that $A_{1} \longrightarrow A_{2}$, by choosing $\pi_{1}$ and $\pi_{2}$ to be appropriate singleton vertices lying in $A_{1} \cap A_{2}$.

We say that ' $A_{1}$ is 2 -connected to $\infty$ ' if there exist two infinite vertex-disjoint paths $\pi_{i}=\left(a_{i, 1}, g_{i, 1}, a_{i, 2}, \ldots\right), i=1,2$, such that $a_{i, 1} \in A_{1}$ for $i=1,2$. In this case, we write $A_{1} \longrightarrow \infty$.

If $A_{1}$ is 2-connected to $A_{2}$ by paths $\pi_{1}, \pi_{2}$ satisfying $V_{\mathrm{h}}\left(\pi_{i}\right) \subseteq \Lambda(M)$ for $i=1,2$, then we write $A_{1} \stackrel{M}{\longrightarrow} A_{2}$.

Lemma 11. Let $x \in \mathbb{Z}^{d}$. There exists a positive integer $I_{x}$, depending on $x$ only, such that $e, f+x . \kappa \subseteq \Lambda\left(I_{x}\right)$ and $e \stackrel{I_{x}}{\longrightarrow} f+x . \kappa$ for all $e, f \in \mathcal{E}$.

Proof. Let $x \in \mathbb{Z}^{d}$. We claim that

$$
e \longrightarrow f+x . \kappa \quad \text { for all } e, f \in \mathcal{E} \text {. }
$$

The lemma is a consequence of (5.3), as the following argument shows. Let $M_{1}$ be such that $e, f+x . \kappa \subseteq \Lambda\left(M_{1}\right)$ for all $e, f \in \mathcal{E}$. If $e \longrightarrow f+x . \kappa$ then there exists a positive integer $M(e, f)$ such that $e \stackrel{M(e, f)}{\longrightarrow} f+x . \kappa$. Now set

$$
M_{2}=\max \{M(e, f): e, f \in \mathcal{E}\},
$$

and $I_{x}=\max \left\{M_{1}, M_{2}\right\}$.

Next we prove (5.3). Let $e, f \in \mathcal{E}$. If $e=f+x . \kappa$ then $|e \cap(f+x . \kappa)| \geq 2$ and the conclusion is immediate; therefore we may assume that $e \neq f+x . \kappa$. Let $F$ be the maximal number of vertex-disjoint paths joining vertices in $e$ to vertices in $f+x$. $\kappa$. Certainly $F \geq 1$ since $\mathbb{H}$ is connected. If $F \geq 2$ then $e \longrightarrow f+x . \kappa$. Suppose then that $F=1$. By Menger's theorem, there exists a vertex $v$ such that every path from $e$ to $f+x . \kappa$ passes through $v$. Since $e$ (respectively $f+x . \kappa)$ is essential, there exist two vertex-disjoint paths $\pi_{1}(e), \pi_{2}(e)$ connecting vertices in $e$ to $\infty$ (respectively $\pi_{1}(f), \pi_{2}(f)$ connecting vertices in $f+x . \kappa$ to $\infty)$.

There exists a path $\nu_{e}$ of minimal length joining $v$ to some vertex of $V\left(\pi_{1}(e)\right) \cup V\left(\pi_{2}(e)\right)$. We pick such a path according to some rule, and may suppose without loss of generality that $\nu_{e}$ has an endpoint in $\pi_{1}(e)$, say $w_{e}$. We pick similarly a path $\nu_{f}$, of minimal length, and joining $v$ to some vertex of $V\left(\pi_{1}(f)\right) \cup V\left(\pi_{2}(f)\right)$; we may suppose that $\nu_{f}$ has an endpoint in $\pi_{1}(f)$, say $w_{f}$. The union of the paths $\pi_{1}(e), \nu_{e}, \nu_{f}, \pi_{1}(f)$ contains a path joining some vertex of $e$ to some vertex of $f+x . \kappa, v i z$., the subpath of $\pi_{1}(e)$ from $e$ to $w_{e}$, followed by $\nu_{e}$ and $\nu_{f}$, followed by the subpath of $\pi_{1}(f)$ joining $w_{f}$ to $f+x$. $\kappa$. Certainly this is an alternating sequence $v_{1}, h_{1}, v_{2}, \ldots, h_{s}, v_{s+1}$ of vertices and hyperedges such that 
$v_{1} \in e, v_{s+1} \in f+x . \kappa$, and $\left\{v_{i}, v_{i+1}\right\} \subseteq h_{i}$ for $1 \leq i \leq s$. If this sequence is not itself a path, then a path may be obtained from it by a process of loop-removal. In this way we obtain a path $\pi$ joining some vertex of $e$ to some vertex of $f+x . \kappa$ which is vertex-disjoint from $\pi_{2}(e)$ and $\pi_{2}(f)$.

Let $N$ be a positive integer such that $V_{\mathrm{h}}(\pi) \subseteq \Lambda(N)$. Since $\pi_{2}(e)$ and $\pi_{2}(f)$ are infinite, they contain (respectively) vertices $x_{e}$ and $x_{f}$ lying outside $\Lambda(N+D)$, where $D$ is given by Lemma 9. Now $x_{e}$ and $x_{f}$ are joined by a path of $\mathbb{Z}^{d}$ (with the usual neighbour-relation) which lies entirely outside $\Lambda(N+D)$. By Lemma 9 , there exists a path $\pi^{\prime}$ of $\mathbb{H}$ joining $x_{e}$ to $x_{f}$ such that $V_{\mathrm{h}}\left(\pi^{\prime}\right) \cap \Lambda(N)=\varnothing$. The union of $\pi_{2}(e), \pi^{\prime}$, and $\pi_{2}(f)$ contains a path $\pi^{\prime \prime}$ of $\mathbb{H}$, joining some vertex of $e$ to some vertex of $f+x . \kappa$, which is vertex-disjoint from $\pi$. However, $v$ does not lie on $\pi^{\prime \prime}$, which contradicts the definition of $v$. It follows that $F \neq 1$, and the proof is complete.

Let $\Lambda$ be a finite box of $\mathbb{Z}^{d}$, let $\widetilde{\mathbf{p}}=\left(p_{e, z}:(e, z) \in \mathbb{E}_{\Lambda}\right)$ be a vector of reals satisfying $0 \leq p_{e, z} \leq 1$, and let $q>0$. From $\widetilde{\mathbf{p}}$ we construct the vector $\widehat{\mathbf{p}}=\left(\widehat{p}_{e, z}:(e, z) \in \mathbb{E}_{\Lambda}\right)$ by

$$
\widehat{p}_{e, z}= \begin{cases}p_{e, z} & \text { if }(e, z) \in \overline{\mathcal{E}} \\ 0 & \text { if }(e, z) \in \overline{\mathcal{I}}\end{cases}
$$

Let $\Omega_{\Lambda}^{\prime}$ be the set of all configurations $\omega^{\prime}=\left(\omega^{\prime}(e, z):(e, z) \in \overline{\mathcal{E}}\right)$ with $\omega^{\prime}(e, z)=1$ if $(e, z) \notin \mathcal{E}_{\Lambda}$, and let $\mathcal{F}_{\Lambda}^{\prime}$ be the $\sigma$-field of all subsets of $\Omega_{\Lambda}^{\prime}$.

Next we define some probability measures. Let $\phi_{\Lambda, \widetilde{\mathbf{p}}, q}$ be the measure on $\left(\Omega_{\Lambda}, \mathcal{F}_{\Lambda}\right)$ given by (4.1), and let $\phi_{\Lambda, \widetilde{\mathbf{p}}, q}^{\prime}$ be the probability measure on $\left(\Omega_{\Lambda}^{\prime}, \mathcal{F}_{\Lambda}^{\prime}\right)$ given by

$$
\phi_{\Lambda, \widetilde{\mathbf{p}}, q}^{\prime}\left(\omega^{\prime}\right)=\frac{1}{Z}\left\{\prod_{(e, z) \in \mathcal{E}_{\Lambda}} p_{e, z}^{\omega^{\prime}(e, z)}\left(1-p_{e, z}\right)^{1-\omega^{\prime}(e, z)}\right\} q^{k\left(\omega^{\prime}\right)}, \quad \omega^{\prime} \in \Omega_{\Lambda}^{\prime} ;
$$

here, $Z$ is the appropriate normalizing factor, and $k\left(\omega^{\prime}\right)$ is the number of components of $\left(V, \eta\left(\omega^{\prime}\right)\right)$, where $\eta\left(\omega^{\prime}\right)=\left\{(e, z) \in \overline{\mathcal{E}}: \omega^{\prime}(e, z)=1\right\}$.

For $\omega \in \Omega_{\Lambda}$, we define $\omega^{\prime}\left(\in \Omega_{\Lambda}^{\prime}\right)$ by

$$
\omega^{\prime}(e, z)=\omega(e, z) \quad \text { if }(e, z) \in \overline{\mathcal{E}} .
$$

For $A^{\prime} \in \mathcal{F}_{\Lambda}^{\prime}$, we define the event $A\left(\in \mathcal{F}_{\Lambda}\right)$ as the set of all $\omega\left(\in \Omega_{\Lambda}\right)$ such that $\omega^{\prime} \in A^{\prime}$; thus, $A$ is the set of all configurations in $\Omega_{\Lambda}$ whose projections onto $\overline{\mathcal{E}}$ lie in $A^{\prime}$, i.e., $A=\left(A^{\prime} \times\{0,1\}^{\mathbb{E} \backslash \overline{\mathcal{E}}}\right) \cap \Omega_{\Lambda}$.

Lemma 12. For any $A^{\prime} \in \mathcal{F}_{\Lambda}^{\prime}$,

$$
\phi_{\Lambda, \widetilde{\mathbf{p}}, q}(A)=\phi_{\Lambda, \widehat{\mathbf{p}}, q}(A)=\phi_{\Lambda, \widetilde{\mathbf{p}}, q}^{\prime}\left(A^{\prime}\right) .
$$

This amounts to saying that, for any event which is defined in terms of the states of the essential elements of $\mathbb{E}_{\Lambda}$ only, this event is independent of the states of the inessential elements.

Proof. Let $A^{\prime} \in \mathcal{F}_{\Lambda}^{\prime}$. It suffices to prove that

$$
\phi_{\Lambda, \widetilde{\mathbf{p}}, q}(A)=\phi_{\Lambda, \widetilde{\mathbf{p}}, q}^{\prime}\left(A^{\prime}\right),
$$


since $\phi_{\Lambda, \widehat{\mathbf{p}}, q}^{\prime}=\phi_{\Lambda, \widetilde{\mathbf{p}}, q}^{\prime}$ by (5.4) and (5.5). Although configurations in $\Omega_{\Lambda}$ and $\Omega_{\Lambda}^{\prime}$ have infinitely many coordinates (since there are infinitely many hyperedges in $\mathbb{E}$ and $\overline{\mathcal{E}}$ ), we may think of $\phi_{\Lambda, \widetilde{\mathbf{p}}, q}$ and $\phi_{\Lambda, \widetilde{\mathbf{p}}, q}^{\prime}$ as being measures on finite hypergraphs derived from $\mathbb{H}$ by identifying all vertices not belonging to $\bar{\Lambda} \backslash \partial \bar{\Lambda}$. To this end we introduce the operator $T_{\Lambda}$ which performs the required identification. That is, for $u \in \mathbb{Z}^{d}$, we define

$$
T_{\Lambda}(u)= \begin{cases}u & \text { if } u \in \bar{\Lambda} \backslash \partial \bar{\Lambda} \\ I & \text { if } u \notin \bar{\Lambda} \backslash \partial \bar{\Lambda}\end{cases}
$$

where $I$ is a title for the composite vertex obtained by such an identification. We write $T_{\Lambda}(e, z)$ for the set of vertices in $T_{\Lambda}(e+z . \kappa)$, where $(e, z) \in \mathbb{E}$, and we denote by $T_{\Lambda} \mathbb{H}$ (respectively $T_{\Lambda} \mathbb{H}_{\mathcal{E}}$ ) the hypergraph obtained from $\mathbb{H}$ (respectively $\mathbb{H}_{\mathcal{E}}$ ) by this identification.

We think of $\phi_{\Lambda, \widetilde{\mathbf{p}}, q}$ as the random-cluster measure on $T_{\Lambda} \mathbb{H}$, and of $\phi_{\Lambda, \widetilde{\mathbf{p}}, q}^{\prime}$ as the randomcluster measure on $T_{\Lambda} \mathbb{H}_{\mathcal{E}}$; this may be done as a consequence of Lemma 10, on observing that, if $\omega \in \Omega_{\Lambda}$, then $\omega(e, z)=1$ for all $(e, z) \notin \mathbb{E}_{\Lambda}$ and $\omega^{\prime}(e, z)=1$ for all $(e, z) \in \overline{\mathcal{E}} \backslash \mathcal{E}_{\Lambda}$.

Let $(e, z)$ be an inessential hyperedge of $\mathbb{E}_{\Lambda}$, i.e., $(e, z) \in \overline{\mathcal{I}} \cap \mathbb{E}_{\Lambda}\left(=\mathbb{E}_{\Lambda} \backslash \mathcal{E}_{\Lambda}\right)$. Making use of Lemma 10 and the discussion prior to Lemma 7 , we have that the hypergraphs $T_{\Lambda} C_{e, z}$ and $T_{\Lambda} D_{e, z}$, where $C_{e, z}=(\bar{g}(e, z), h(e, z))$ and $D_{e, z}=\left(\mathbb{Z}^{d} \backslash g(e, z), \mathbb{E} \backslash h(e, z)\right)$, have a unique vertex in common (viz. $T_{\Lambda}(c(e, z))$ ). Furthermore, $T_{\Lambda} \mathbb{H}$ may be expressed as the union $T_{\Lambda} C_{e, z} \cup T_{\Lambda} D_{e, z}$ of two hypergraphs having exactly one vertex in common. In addition, the event $A^{\prime}$ is defined in terms of the states of essential hyperedges only, each of which has image (under $T_{\Lambda}$ ) belonging to $T_{\Lambda} D_{e, z}$. It follows from Lemma 6 that $A^{\prime}$ is independent of all hyperedge-states in $C_{e, z}$, which is to say that

$$
\phi_{\Lambda, \widetilde{\mathbf{p}}, q}(A)=\widehat{\phi}_{e, z}\left(A_{e, z}\right)
$$

where $\widehat{\phi}_{e, z}$ is the random-cluster measure defined as was $\phi_{\Lambda, \widetilde{\mathbf{p}}, q}$ but on the smaller hypergraph $D_{e, z}$, and $A_{e, z}$ is the set of all configurations in $\{0,1\}^{\mathbb{E} \backslash h(e, z)}$ whose projections onto $\overline{\mathcal{E}}$ lie in $A^{\prime}$

Having dealt with the inessential hyperedge $(e, z)$ and the associated region $(\bar{g}(e, z)$, $h(e, z))$, we pick another inessential element of $\mathbb{E}_{\Lambda}$, and iterate the argument. Once all the inessential elements of $\mathbb{E}_{\Lambda}$ have been removed, one obtains (5.7) in like manner as (5.8).

\section{Remaining proofs}

Proof of Theorem 1. (a) Let $A(\in \mathcal{F})$ be the event $\{0 \leftrightarrow \infty\}$. By Lemmas 7 and 8 , the states of inessential hyperedges are irrelevant to the occurrence or non-occurrence of $A$, and therefore

$$
A=A^{\prime} \times\{0,1\}^{\mathbb{E} \backslash \overline{\mathcal{E}}}
$$

where $A^{\prime}\left(\subseteq\{0,1\}^{\overline{\mathcal{E}}}\right)$ is the set of configurations of essential hyperedges in which there exists an infinite path beginning at the origin.

We may apply Lemma 12 to the event $A^{\prime} \cap \Omega_{\Lambda}^{\prime}\left(\in \mathcal{F}_{\Lambda}^{\prime}\right)$ to deduce that

$$
\phi_{\Lambda, \widetilde{\mathbf{p}}, q}\left(A \cap \Omega_{\Lambda}\right)=\phi_{\Lambda, \widehat{\mathbf{p}}, q}\left(A \cap \Omega_{\Lambda}\right)=\phi_{\Lambda, \widetilde{\mathbf{p}}, q}^{\prime}\left(A^{\prime} \cap \Omega_{\Lambda}^{\prime}\right),
$$


in the notation of that lemma. Therefore

$$
\theta_{\Lambda}(\mathbf{p}, q)=\theta_{\Lambda}(\widehat{\mathbf{p}}, q)
$$

in the notation of Theorem 1. The claim of part (a) follows in the limit as $\Lambda \uparrow \mathbb{Z}^{d}$.

(b) Let $q>1$. Let $\mathbf{p} \in[0,1]^{L}$ be such that $p_{e}>0$ for all $e \in \mathcal{E}$, and let $\theta_{\Lambda}^{\prime}(\mathbf{p}, q)=\phi_{\Lambda, \widetilde{\mathbf{p}}, q}^{\prime}\left(A^{\prime}\right)$ where $\widetilde{\mathbf{p}}$ is given by $p_{e, z}=p_{e}$, and $A^{\prime}$ is defined above. We have, by (6.2), that

$$
\theta_{\Lambda}(\mathbf{p}, q)=\theta_{\Lambda}^{\prime}(\mathbf{p}, q)
$$

and therefore

$$
\theta^{\prime}(\mathbf{p}, q)=\lim _{\Lambda \uparrow \mathbb{Z}^{d}} \theta_{\Lambda}^{\prime}(\mathbf{p}, q)
$$

exists and satisfies

$$
\theta^{\prime}(\mathbf{p}, q)=\theta(\mathbf{p}, q)
$$

Once Theorem 2 has been proved, it will follow from (6.4) that there exists a positive integer $N$ and a continuous function $\alpha:(0,1)^{\mathcal{E}} \rightarrow(0, \infty)$ such that $\theta_{n}^{\prime}(\mathbf{p}, q)=\theta_{\Lambda(n)}^{\prime}(\mathbf{p}, q)$ satisfies

$$
\frac{\partial \theta_{n}^{\prime}}{\partial p_{e}} \leq \alpha(\mathbf{p}) \frac{\partial \theta_{n}^{\prime}}{\partial p_{f}}
$$

for all $n \geq N$ and all $e, f \in \mathcal{E}$. We now follow the proof of Theorem 2 of [BGK] exactly, to deduce that there exist positive constants $\delta, \nu$, and $\epsilon_{0}$, together with a full subset $F$ of $\mathbb{R}^{L}$, such that

$$
\theta^{\prime}(\mathbf{p}+\nu \epsilon \mathbf{g}, q) \leq \theta^{\prime}(\mathbf{p}+\epsilon \mathbf{h}, q) \leq \theta^{\prime}(\mathbf{p}+\delta \epsilon \mathbf{g}, q)
$$

for all $0<\epsilon<\epsilon_{0}$ and all $\mathbf{g}, \mathbf{h} \in F$. Inequality (3.9) is a consequence of this, by applying (6.5).

Proof of Theorem 2. It is a consequence of (3.8) that the probability of the event $A=$ $\{0 \leftrightarrow \infty\}$ is unchanged by setting $p_{e}=0$ for all hyperedges $e$ such that $(e, 0)$ is inessential. As is implicit in Lemma 12, this amounts to working with the connected hypergraph $\mathbb{H}_{\mathcal{E}}$ containing the essential hyperedges only, rather than working with the original hypergraph $\mathbb{H}$. We shall find it slightly more convenient to work instead on $\mathbb{H}$ with $p_{e}$ set to some fixed value for $e \in \mathcal{I}$, say $p_{e}=\frac{1}{2}$. Such values are immaterial to the probability of $A$ by virtue of (3.8).

Let $\mathbf{p} \in(0,1)^{L}$ and suppose $p_{e}=\frac{1}{2}$ for $e \in \mathcal{I}$. Let

$$
\pi(\mathbf{p})=\min \left\{p_{e}, 1-p_{e}: e \in \mathcal{E}\right\},
$$

and let $\widetilde{\mathbf{p}}=\left(p_{e, z}:(e, z) \in \mathbb{E}\right)$ satisfy

$$
\frac{1}{2} \pi(\mathbf{p})<p_{e, z}<1-\frac{1}{2} \pi(\mathbf{p}) \quad \text { for }(e, z) \in \overline{\mathcal{E}},
$$

and $p_{e, z}=p_{e}=\frac{1}{2}$ for $(e, z) \in \overline{\mathcal{I}}$. Let $n$ be a positive integer, and let $\phi_{n}$ be the measure defined in (4.1) with $\Lambda=\Lambda(n)$. 
We have that

$$
\frac{\partial \theta_{n}}{\partial p_{e}}=\left.\sum_{z:(e, z) \in \mathcal{E}_{\Lambda(n)}} \frac{\partial \theta_{n}}{\partial p_{e, z}}\right|_{\widetilde{\mathbf{p}}=\mathbf{p}}, \quad e \in \mathcal{E},
$$

where $\theta_{n}(\widetilde{\mathbf{p}}, q)=\phi_{n}(A)$. In writing $\widetilde{\mathbf{p}}=\mathbf{p}$ we mean the second derivative to be evaluated by setting $p_{e, z}=p_{e}$ for all $(e, z) \in \mathcal{E}_{\Lambda(n)}$.

Let $(e, z),(f, x) \in \mathcal{E}_{\Lambda(n)}$, and let $M$ be a positive integer. We write $(e, z) \stackrel{M, n}{\longrightarrow}(f, x)$ if there exist two paths $\pi_{1}$ and $\pi_{2}$ of $\mathbb{H}$, each joining a vertex of $e+z . \kappa$ to a vertex of $f+x . \kappa$, such that

(a) these paths are vertex-disjoint,

(b) $V_{\mathrm{h}}\left(\pi_{i}\right) \cap \overline{\Lambda(n)} \subseteq \Lambda(M)+z . \kappa$ for $i=1,2$, and also $e+z . \kappa, f+x . \kappa \subseteq \Lambda(M)+z . \kappa$,

(c) no more than one of $\pi_{1}, \pi_{2}$ contains vertices of $\partial \overline{\Lambda(n)}$.

If (a), (b), and (c) are satisfied, it is a consequence of Lemma 7 that every hyperedge of $\pi_{1}$ and $\pi_{2}$ is essential, and in addition at least one of $\pi_{1}, \pi_{2}$ uses only hyperedges in $\mathcal{E}_{\Lambda(n)}$. We write $(e, z) \stackrel{M, n}{\Longleftrightarrow}(f, x)$ if two such paths $\pi_{1}, \pi_{2}$ may be found which satisfy $V_{\mathrm{h}}\left(\pi_{1}\right) \cap V_{\mathrm{h}}\left(\pi_{2}\right)=\varnothing$ in addition.

The principal steps in the proof of Theorem 2 are contained in the next two lemmas.

Lemma 13. Let $M$ be a positive integer. There exists a continuous function $\alpha_{M}$ : $(0,1)^{L} \rightarrow(0, \infty)$ such that

$$
\frac{\partial \theta_{n}}{\partial p_{e, z}} \leq \alpha_{M}(\mathbf{p}) \frac{\partial \theta_{n}}{\partial p_{f, x}}
$$

for all $(e, z),(f, x) \in \mathcal{E}_{\Lambda(n)}$ such that $(e, z) \stackrel{M, n}{\Longleftrightarrow}(f, x)$.

Proof. This fundamental lemma is proved using Proposition 5 in a manner closely related to the proof of Theorem 1 of [BGK].

Take $A=\{0 \leftrightarrow \infty\}$ as usual, so that $\theta_{n}(\widetilde{\mathbf{p}}, q)=\phi_{n}(A)$. Let $(X, Y)$ be the Markov chain constructed in Section 4 for this event, using the region $\Lambda=\Lambda(n)$. Suppose that $(e, z)$ and $(f, x)$ satisfy the condition of the lemma, and let $\pi_{1}=\left(u_{1}, a_{1}, u_{2}, \ldots, a_{r-1}, u_{r}\right)$ and $\pi_{2}=\left(v_{1}, b_{1}, v_{2}, \ldots, b_{s-1}, v_{s}\right)$ be paths satisfying (a)-(c) above, and with $u_{1}, v_{1} \in e+z . \kappa$, $u_{r}, v_{s} \in f+x . \kappa$, and $V_{\mathrm{h}}\left(\pi_{1}\right) \cap V_{\mathrm{h}}\left(\pi_{2}\right)=\varnothing$; by (c), we may assume that $\pi_{1}$ contains no vertex of $\partial \overline{\Lambda(n)}$, and hence $a_{i} \in \mathcal{E}_{\Lambda(n)}$ for all $i$. See Figure 3 .

By Proposition 5,

$$
\frac{\partial \theta_{n}}{\partial p_{e, z}} / \frac{\partial \theta_{n}}{\partial p_{f, x}}=\gamma(\widetilde{\mathbf{p}}) \frac{\lim _{t \rightarrow \infty}\left\{P\left(X_{t}(e, z)=0, Y_{t}(e, z)=1\right)\right\}}{\lim _{t \rightarrow \infty}\left\{P\left(X_{t}(f, x)=0, Y_{t}(f, x)=1\right)\right\}}
$$

for some continuous $\gamma$ which is positive and finite when $\mathbf{0}<\widetilde{\mathbf{p}}<\mathbf{1}$. We shall show that there exists $\nu(\widetilde{\mathbf{p}})$, continuous and strictly positive when $\mathbf{0}<\widetilde{\mathbf{p}}<\mathbf{1}$, such that

$$
P\left(X_{t+3}(f, x)=0, Y_{t+3}(f, x)=1\right) \geq \nu(\widetilde{\mathbf{p}}) P\left(X_{t}(e, z)=0, Y_{t}(e, z)=1\right) \quad \text { for all } t .
$$

This clearly implies that

$$
\frac{\lim _{t \rightarrow \infty}\left\{P\left(X_{t}(e, z)=0, Y_{t}(e, z)=1\right)\right\}}{\lim _{t \rightarrow \infty}\left\{P\left(X_{t}(f, x)=0, Y_{t}(f, x)=1\right)\right\}} \leq \frac{1}{\nu(\widetilde{\mathbf{p}})},
$$


which in turn yields (6.11) with some $\alpha_{M}$ satisfying $\alpha_{M}(\mathbf{p}) \geq \gamma(\widetilde{\mathbf{p}}) / \nu(\widetilde{\mathbf{p}})$, via (6.9) and (6.12). Inequality (6.13) will be obtained by showing that

$$
P\left(X_{t+3}(f, x)=0, Y_{t+3}(f, x)=1 \mid X_{t}(e, z)=0, Y_{t}(e, z)=1\right) \geq \nu(\widetilde{\mathbf{p}}) .
$$

We now prove (6.15). Let $B_{z}$ be the collection of all hyperedges of $\mathbb{H}$ which are subsets of $(z . \kappa+\Lambda(M)) \cap \overline{\Lambda(n)}$; these are hyperedges which are 'near' to $e+z . \kappa$ and lie in $\overline{\Lambda(n)}$. In particular, $(e, z),(f, x) \in B_{z}$, and in addition $B_{z}$ contains all hyperedges of $\pi_{1}$ and $\pi_{2}$ which belong to $\mathbb{E}_{\Lambda(n)}$. Therefore $a_{i} \in B_{z}$ for all $i$. We shall say that a hyperedge $(g, y)$ is 'present in the process $Z$ at time $s$ ' if $Z_{s}(g, y)=1$. We introduce the following events:

$$
\begin{aligned}
V_{t} & =\left\{X_{t}(e, z)=0, Y_{t}(e, z)=1\right\}, \\
W_{t} & =\left\{X_{t}(f, x)=0, Y_{t}(f, x)=1\right\} .
\end{aligned}
$$

In addition we define the events $V^{i}, i=1,2,3$, by

(i) $V^{1}$ is the event that: during the time-interval $(t, t+1]$, all hyperedges in $B_{z}$ which are present in $X_{t}$ are removed, and no hyperedges in $B_{z}$ are added to $X ;(e, z)$ remains present in $Y$,

(ii) $V^{2}$ is the event that: during $(t+1, t+2]$, the hyperedges $a_{1}, a_{2}, \ldots, a_{r-1}, b_{1}, b_{2}, \ldots$, $b_{s-1},(f, x)$ are added to $X$, but no other hyperedges in $B_{z}$ are added to $X ;(e, z)$ remains present in $Y$,

(iii) $V^{3}$ is the event that: during $(t+2, t+3]$, the hyperedge $(f, x)$ is removed from $X$ but not from $Y$.

Note that, due to the coupling of $X$ and $Y$, the occurrence of $V^{1}$ may force the removal of some hyperedges from $Y$ during $(t, t+1]$. However, on $V_{t},(e, z)$ is absent from $X$ and present in $Y$ at time $t$. Thus, for $V^{1}$ to occur, $X_{s}(e, z)$ and $Y_{s}(e, z)$ must remain constant for $t<s \leq t+1$. Similarly, the occurrence of $V^{2}$ will force the addition of $a_{1}, a_{2}, \ldots, a_{r-1}, b_{1}, b_{2}, \ldots, b_{s-1},(f, x)$ to $Y$, if not already present at time $t+1$, because $X_{s} \leq Y_{s}$ (see also (4.11)-(4.13)). If any of the hyperedges $a_{1}, a_{2}, \ldots, a_{r-1}, b_{1}, b_{2}, \ldots, b_{s-1}$ lie outside $\mathbb{E}_{\Lambda(n)}$, then it is automatically present at all times in both $X$ and $Y$, and the requirement on such a hyperedge under $V^{2}$ is vacuous. Note that none of the events $V_{t}$, $V^{i}$ for $1 \leq i \leq 3$, places any constraint on the states of hyperedges outside $B_{z}$.

Clearly $V_{t} \cap V^{1} \cap V^{2} \cap V^{3} \subseteq W_{t+3}$. In order to prove (6.15) it therefore suffices to show that

$$
P\left(V^{1} \cap V^{2} \cap V^{3} \mid V_{t}\right) \geq \nu(\widetilde{\mathbf{p}})
$$

Assume now that $V_{t}$ occurs. According to (4.10) and (4.11), each hyperedge $(g, y)$ $\left(\in \mathbb{E}_{\Lambda(n)}\right)$ that is present in $X$ is lost at a rate which is bounded away from 0 , uniformly in the states of all other edges (and uniformly in all $\widetilde{\mathbf{p}}$ satisfying (6.9)). Moreover, the total rate at which changes occur within $B_{z}$ is no larger than the following upper bound for the sum of birth and death rates,

$$
\left|B_{z}\right|\left(1+2 q^{\mu} \max \left\{\frac{1-p_{g, y}}{p_{g, y}}:(g, y) \in \mathbb{E}_{\Lambda(n)}\right\}\right) \leq\left|B_{z}\right|\left(1+2 q^{\mu} \frac{2-\pi(\mathbf{p})}{\pi(\mathbf{p})}\right)=U
$$


by (4.6)-(4.11), where $\mu=\max \{|e|: e \in K\}-1$; the bound $U$ does not depend on $n$ or $z$ but only on $M$ and $\mathbf{p}$. Therefore there exists $\nu_{1}(\widetilde{\mathbf{p}})$ such that

$$
P\left(V^{1} \mid V_{t}\right) \geq \nu_{1}(\widetilde{\mathbf{p}})>0
$$

the quantity $\nu_{1}(\widetilde{\mathbf{p}})$ (and quantities $\nu_{i}(\widetilde{\mathbf{p}})$ to be defined soon) may be chosen to be continuous and strictly positive when $\mathbf{0}<\widetilde{\mathbf{p}}<\mathbf{1}$, and not to depend on the values of $n$ and $z$.

Underlying the argument of the previous paragraph is a small lemma concerning Markov chains, which we choose not to state formally. Such a lemma amounts roughly to the following. If, for each hyperedge $(g, y)$ belonging to some set $\mathcal{G}$ of bounded cardinality, the jump-rate of some transition of the state of $(g, y)$ is bounded away from 0 , uniformly in the current states of all other hyperedges, then the probability that the appropriate transitions take place on all the hyperedges in $\mathcal{G}$ during a given time-interval is uniformly bounded away from 0. Similarly, if the total jump-rate on a collection $\mathcal{G}^{\prime}$ of hyperedges is uniformly bounded away from $\infty$, then the probability of any change at all on $\mathcal{G}^{\prime}$ is uniformly bounded away from 1 . A simple way of seeing this is to construct the chain in terms of 'exponential alarm clocks' sitting on the different hyperedges of $\mathcal{G}$ and $\mathcal{G}^{\prime}$, so that each hyperedge examines its state whenever its alarm clock rings, and it changes its current state according to a probability distribution which may be a function of the current states of the other hyperedges.

Suppose that the event $V_{t} \cap V^{1}$ occurs, and consider the event $V^{2}$. Each hyperedge $(g, y)\left(\in B_{z}\right)$ is acquired by $X$ (and also by $Y$ if not already present there) at rate 1 (see (4.9)); therefore there exists $\nu_{2}(\widetilde{\mathbf{p}})$ of the required form such that

$$
P\left(V^{2} \mid V_{t} \cap V^{1}\right) \geq \nu_{2}(\widetilde{\mathbf{p}})>0 .
$$

Suppose that $V_{t} \cap V^{1} \cap V^{2}$ occurs, and consider $V^{3}$. At time $t+2$, the only hyperedges of $B_{z}$ which are present in $X$ are $(f, x)$ together with any $a_{i}$ and $b_{j}$ belonging to $\mathbb{E}_{\Lambda(n)}$. In general $Y_{t+2}$ contains more hyperedges than does $X_{t+2}$, and certainly at least $(e, z), a_{1}, a_{2}, \ldots, a_{r-1}, b_{1}, b_{2}, \ldots, b_{s-1},(f, x)$. We have therefore that $D_{f, x}\left(X_{t+2}\right)>$ $D_{f, x}\left(Y_{t+2}\right)$; see Figure 3 and remember that $V_{\mathrm{h}}\left(\pi_{1}\right) \cap V_{\mathrm{h}}\left(\pi_{2}\right)=\varnothing$. It follows by (4.11)(4.13) that the rate at which $(f, x)$ is removed from $Y$ but not from $X$ is at least

$$
\frac{1-p_{f, x}}{p_{f, x}}(q-1) \geq \frac{\pi(\mathbf{p})}{2-\pi(\mathbf{p})}(q-1)>0 .
$$

This implies that

$$
P\left(V^{3} \mid V_{t} \cap V^{1} \cap V^{2}\right) \geq \nu_{3}(\widetilde{\mathbf{p}})>0
$$

for some suitable $\nu_{3}(\widetilde{\mathbf{p}})$. This in turn implies $\left(6\right.$.15) with $\nu(\widetilde{\mathbf{p}})=\nu_{1}(\widetilde{\mathbf{p}}) \nu_{2}(\widetilde{\mathbf{p}}) \nu_{3}(\widetilde{\mathbf{p}})$; as described before, (6.11) follows for a suitable function $\alpha_{M}$.

Lemma 14. Let $M$ be a positive integer. There exists a continuous function $\beta_{M}$ : $(0,1)^{L} \rightarrow(0, \infty)$ such that

$$
\frac{\partial \theta_{n}}{\partial p_{e, z}} \leq \beta_{M}(\mathbf{p}) \frac{\partial \theta_{n}}{\partial p_{f, x}}
$$


for all $(e, z),(f, x) \in \mathcal{E}_{\Lambda(n)}$ such that $(e, z) \stackrel{M, n}{\longrightarrow}(f, x)$.

Proof. We begin with a graph-theoretic observation. Let $\pi=\left(v_{1}, e_{1}, v_{2}, \ldots, e_{r}, v_{r+1}\right)$ be a path of $\mathbb{H}$. By the definition of a path, the $v_{i}$ and $e_{j}$ are distinct, but there may exist $v_{i}$ and $e_{j}$ such that $v_{i} \in e_{j}$ but $j \notin\{i-1, i\}$ (if no such $v_{i}, e_{j}$ exist, we call $\pi$ loop-free). However, there exists a path $\pi^{\prime \prime}=\left(u_{1}=v_{1}, f_{1}, u_{2}, \ldots, f_{s}, u_{s+1}=v_{r+1}\right)$ obtained from $\pi$ by deleting vertices and hyperedges, which joins $v_{1}$ to $v_{r+1}$ and has the further property that $u_{i} \in f_{j}$ if and only if $j \in\{i-1, i\}$. Such a path $\pi^{\prime \prime}$ is obtained from $\pi$ by a process of loop-removal, as follows. Let $J=J(\pi)$ and $K=K(\pi)$ be given by

$$
\begin{aligned}
J & =\min \left\{j: v_{j} \in e_{k} \text { for some } k>j\right\}, \\
K & =\min \left\{k: v_{j} \in e_{k} \text { for some } j>k+1\right\},
\end{aligned}
$$

with the convention that the minimum of the empty set is $\infty$. If $J \leq K$ and $J<\infty$, we remove from $\pi$ the sequence $e_{J}, v_{J+1}, \ldots, v_{J^{\prime}}$ where

$$
J^{\prime}=\max \left\{l: v_{J} \in e_{l}\right\}
$$

note that $J^{\prime}<r+1$, so that $v_{J^{\prime}} \neq v_{r+1}$. If $K<J$ (and so $K<\infty$, also), we remove from $\pi$ the sequence $v_{K+1}, e_{K+1}, \ldots, e_{K^{\prime}-1}$ where

$$
K^{\prime}=\max \left\{m: v_{m} \in e_{K}\right\}
$$

note that $K+1 \geq 2$, so that $v_{K+1} \neq v_{1}$. This results in a new path $\pi^{\prime}$ which is strictly shorter than $\pi$ whenever either $J(\pi)$ or $K(\pi)$ is finite. We iterate this construction, obtaining thus a sequence $\pi, \pi^{\prime}, \ldots, \pi^{\prime \prime}$ of paths, where $\pi^{\prime \prime}$ joins $v_{1}$ to $v_{r+1}$ and satisfies $J\left(\pi^{\prime \prime}\right), K\left(\pi^{\prime \prime}\right)=\infty ; \pi^{\prime \prime}$ is the required path.

Next, we describe a construction on pairs of paths. Let $(h, x) \in \mathbb{E}$. Suppose that $\left(\pi_{1}, \pi_{2}\right)$ is a pair of finite vertex-disjoint paths of $\mathbb{H}$, say $\pi_{i}=\left(a_{i, 1}, g_{i, 1}, a_{i, 2}, \ldots, g_{i, r_{i}}, a_{i, r_{i}+1}\right)$ where the $a_{i, j}$ are vertices and the $g_{i, j}$ are hyperedges. We assume that the triple $(h, x), \pi_{1}, \pi_{2}$ is such that $a_{i, 1} \in h+x . \kappa$ but $a_{i, j} \notin h+x . \kappa$ for $i=1,2$ and $j \geq 2$. We assume in addition that $\pi_{1}$ and $\pi_{2}$ have the property described above, i.e.,

$$
a_{i, j} \in g_{i, k} \text { if and only if } k \in\{j-1, j\} \text {, for } i=1,2 \text {. }
$$

We shall describe a method which replaces the pair $\left(\pi_{1}, \pi_{2}\right)$ by two pairs $\left(\sigma_{1}, \sigma_{2}\right)$ and $\left(\pi_{1}^{\prime}, \pi_{2}^{\prime}\right)$ of paths, where $\sigma_{i}$ and $\pi_{i}^{\prime}$ are (apart from minor changes) subpaths of $\pi_{i}$, for $i=1,2$.

Let

$$
A_{1}=\min \left\{j: g_{1, j} \cap V_{\mathrm{h}}\left(\pi_{2}\right) \neq \varnothing, 1 \leq j \leq r_{1}\right\} ;
$$

if no such $j$ exists, then $V_{\mathrm{h}}\left(\pi_{1}\right) \cap V_{\mathrm{h}}\left(\pi_{2}\right)=\varnothing$ and we shall do nothing. Assume then that $1 \leq A_{1} \leq r_{1}$, and let

$$
\begin{aligned}
& A_{2}=\min \left\{k: g_{1, A_{1}} \cap g_{2, k} \neq \varnothing, 1 \leq k \leq r_{2}\right\}, \\
& B_{1}=\max \left\{j: a_{1, j} \in g_{2, A_{2}}, 1 \leq j \leq r_{1}\right\}, \\
& B_{2}=\max \left\{k: a_{2, k} \in g_{1, A_{1}}, 1 \leq k \leq r_{2}\right\},
\end{aligned}
$$


whenever these two maxima exist. In order to simplify the notation a little, we write

$$
\begin{aligned}
& \alpha_{1}=a_{1, A_{1}}, \beta_{1}=a_{1, A_{1}+1}, \gamma_{1}=a_{1, B_{1}}, e_{1}=g_{1, A_{1}}, \\
& \alpha_{2}=a_{2, A_{2}}, \beta_{2}=a_{2, A_{2}+1}, \gamma_{2}=a_{2, B_{2}}, e_{2}=g_{2, A_{2}} .
\end{aligned}
$$

Suppose first that $1<A_{i} \leq r_{i}$ for $i=1,2$; there is an extra complication when $A_{i}=1$ for some $i$, and we shall deal with this later. Since $A_{i}>1$ for $i=1,2$, it follows from the definition of the $A_{i}$ that

$$
\alpha_{1} \notin e_{2}, \alpha_{2} \notin e_{1} .
$$

There are several cases, illustrated in Figure 4.

Case $A$. If $\beta_{2} \in e_{1}$, then $A_{2}+1 \leq B_{2} \leq r_{2}$ and we define

$$
\begin{aligned}
\sigma_{1} & =\left(a_{1,1}, g_{1,1}, \ldots, g_{1, A_{1}-1}, \alpha_{1}\right), \\
\sigma_{2} & =\left(a_{2,1}, g_{2,1}, \ldots, a_{2, A_{2}-1}, \alpha_{2}, e_{2}, \beta_{2}\right), \\
\pi_{1}^{\prime} & =\left(\beta_{1}, g_{1, A_{1}+1}, \ldots, g_{1, r_{1}}, a_{1, r_{1}+1}\right), \\
\pi_{2}^{\prime} & =\left(\gamma_{2}, g_{2, B_{2}}, \ldots, g_{2, r_{2}}, a_{2, r_{2}+1}\right), \\
\left(h^{\prime}, x^{\prime}\right) & =e_{1}\left(=g_{1, A_{1}}\right) .
\end{aligned}
$$

Case $B$. If $\beta_{2} \notin e_{1}$ but $\beta_{1} \in e_{2}$, then $A_{1}+1 \leq B_{1} \leq r_{1}$ and we define

$$
\begin{aligned}
\sigma_{1} & =\left(a_{1,1}, g_{1,1}, \ldots, g_{1, A_{1}-1}, \alpha_{1}, e_{1}, \beta_{1}\right), \\
\sigma_{2} & =\left(a_{2,1}, g_{2,1}, \ldots, g_{2, A_{2}-1}, \alpha_{2}\right), \\
\pi_{1}^{\prime} & =\left(\gamma_{1}, g_{1, B_{1}}, \ldots, g_{1, r_{1}}, a_{1, r_{1}+1}\right), \\
\pi_{2}^{\prime} & =\left(\beta_{2}, g_{2, A_{2}+1}, \ldots, g_{2, r_{2}}, a_{2, r_{2}+1}\right), \\
\left(h^{\prime}, x^{\prime}\right) & =e_{2}\left(=g_{2, A_{2}}\right) .
\end{aligned}
$$

Case $C$. If $\beta_{2} \notin e_{1}$ and $\beta_{1} \notin e_{2}$, we pick a vertex $w$ satisfying $w \in e_{1} \cap e_{2}$ (note that $w \notin V\left(\pi_{1}\right) \cap V\left(\pi_{2}\right)$ by $\left.(6.23)\right)$ and define

$$
\begin{aligned}
\sigma_{1} & =\left(a_{1,1}, g_{1,1}, \ldots, g_{1, A_{1}-1}, \alpha_{1}\right), \\
\sigma_{2} & =\left(a_{2,1}, g_{2,1}, \ldots, a_{2, A_{2}-1}, \alpha_{2}, e_{2}, w\right), \\
\pi_{1}^{\prime} & =\left(\beta_{1}, g_{1, A_{1}+1}, \ldots, g_{1, r_{1}}, a_{1, r_{1}+1}\right), \\
\pi_{2}^{\prime} & =\left(w, e_{2}, \beta_{2}, g_{2, A_{2}+1}, \ldots, g_{2, r_{2}}, a_{2, r_{2}+1}\right), \\
\left(h^{\prime}, x^{\prime}\right) & =e_{1}\left(=g_{1, A_{1}}\right) .
\end{aligned}
$$

Suppose now that $A_{1}=1$ but $A_{2}>1$. In this case it may occur that $\alpha_{1} \in e_{2}$. If $\alpha_{1} \notin e_{2}$, we act as above. On the other hand:

Case D. If $\alpha_{1} \in e_{2}$, then $1 \leq B_{1} \leq r_{1}$ and we define

$$
\begin{aligned}
\sigma_{1} & =\left(\alpha_{1}\right), \\
\sigma_{2} & =\left(a_{2,1}, g_{2,1}, \ldots, g_{2, A_{2}-1}, \alpha_{2}\right), \\
\pi_{1}^{\prime} & =\left(\gamma_{1}, g_{1, B_{1}}, \ldots, g_{1, r_{1}}, a_{1, r_{1}+1}\right), \\
\pi_{2}^{\prime} & =\left(\beta_{2}, g_{2, A_{2}+1}, \ldots, g_{2, r_{2}}, a_{2, r_{2}+1}\right), \\
\left(h^{\prime}, x^{\prime}\right) & =e_{2}\left(=g_{2, A_{2}}\right) .
\end{aligned}
$$


Suppose that $A_{1}>1$ but $A_{2}=1$. If $\alpha_{2} \notin e_{1}$, we act as in Cases A, B, C, above. On the other hand:

Case $E$. If $\alpha_{2} \in e_{1}$, then $1 \leq B_{2} \leq r_{2}$ and we define

$$
\begin{aligned}
\sigma_{1} & =\left(a_{1,1}, g_{1,1}, \ldots, g_{1, A_{1}-1}, \alpha_{1}\right), \\
\sigma_{2} & =\left(\alpha_{2}\right) \\
\pi_{1}^{\prime} & =\left(\beta_{1}, g_{1, A_{1}+1}, \ldots, g_{1, r_{1}}, a_{1, r_{1}+1}\right), \\
\pi_{2}^{\prime} & =\left(\gamma_{2}, g_{2, B_{2}}, \ldots, g_{2, r_{2}}, a_{2, r_{2}+1}\right), \\
\left(h^{\prime}, x^{\prime}\right) & =e_{1}\left(=g_{1, A_{1}}\right) .
\end{aligned}
$$

Finally suppose that $A_{1}=A_{2}=1$. If $\alpha_{1} \notin e_{2}$ and $\alpha_{2} \notin e_{1}$, we act according to Cases $\mathrm{A}, \mathrm{B}$, and C. If $\alpha_{1} \in e_{2}$ we act according to Case $\mathrm{D}$, and if $\alpha_{1} \notin e_{2}$ but $\alpha_{2} \in e_{1}$ we act according to Case E.

We do one more thing before continuing. Whenever Case $\mathrm{C}$ is invoked, a new vertex $w$ is involved. If (under this case) $w$ lies in some hyperedge of $\pi_{2}^{\prime}$ (given in Case C) in addition to $e_{2}$, then the new path $\pi_{2}^{\prime}$ is not loop-free. If this occurs, we replace $\pi_{2}^{\prime}$ by a new path obtained from $\pi_{2}^{\prime}$ by the process of loop-removal described at the beginning of this proof. Rather than introduce more notation, we shall continue to denote this new path by $\pi_{2}^{\prime}$.

By way of outcome, we obtain a family of objects which we write as $\left\{(h, x), \sigma_{1}, \sigma_{2}\right.$, $\left.\left(h^{\prime}, x^{\prime}\right), \pi_{1}^{\prime}, \pi_{2}^{\prime}\right\}$, and the following may be checked in each of the above cases.

(i) $\sigma_{1}$ and $\sigma_{2}$ are vertex-disjoint paths having initial endpoints in $h+x . \kappa$ and final endpoints in $h^{\prime}+x^{\prime} . \kappa$, and such that $V_{\mathrm{h}}\left(\sigma_{1}\right) \cap V_{\mathrm{h}}\left(\sigma_{2}\right)=\varnothing ; \sigma_{i}$ is a subpath of $\pi_{i}$ for $i=1,2$.

(ii) $\pi_{1}^{\prime}$ and $\pi_{2}^{\prime}$ are vertex-disjoint paths having initial endpoints in $h^{\prime}+x^{\prime} . \kappa$ and having the same final endpoints as $\pi_{1}$ and $\pi_{2}$. In addition, $\pi_{1}^{\prime}$ is a subpath of $\pi_{1}$, and every hyperedge of $\pi_{2}^{\prime}$ lies in $\pi_{2}$. The $\pi_{i}^{\prime}$ have no loops; $\pi_{i}^{\prime}$ is no longer than $\pi_{i}$ for $i=1,2$, and is strictly shorter for at least one value of $i$.

(iii) $\left(h^{\prime}, x^{\prime}\right)$ contains a vertex of $\pi_{1}$, and lies in either $\pi_{1}$ or $\pi_{2}$.

Let $(e, z),(f, x)$ satisfy the condition of the lemma. Let $\pi_{i}=\left(u_{i}=a_{i, 1}, g_{i, 1}, a_{i, 2}, \ldots\right.$, $\left.g_{i, r_{i}}, a_{i, r_{i}+1}=v_{i}\right), i=1,2$, be vertex-disjoint paths with $u_{i} \in e+z . \kappa, v_{i} \in f+x . \kappa$, $V_{\mathrm{h}}\left(\pi_{i}\right) \cap \overline{\Lambda(n)} \subseteq z . \kappa+\Lambda(M)$, for $i=1,2$, and assume that $\pi_{1}$ contains no vertices of $\partial \overline{\Lambda(n)}$. We may assume that $a_{i, j} \neq(e, z),(f, x)$ for all $i, j$; if this fails, we replace $\pi_{1}$ and $\pi_{2}$ by appropriate subpaths. Without loss of generality, we may suppose that (6.23) holds; if (6.23) fails, then we replace $\left(\pi_{1}, \pi_{2}\right)$ by subpaths obtained by the process of loop-removal. Let $H^{\prime}=\left\{j: g_{1, j} \cap V_{\mathrm{h}}\left(\pi_{2}\right) \neq \varnothing\right\}$ be the set of indices of hyperedges in $\pi_{1}$ which intersect hyperedges lying in $\pi_{2}$.

If $H^{\prime}=\varnothing$, then $V_{\mathrm{h}}\left(\pi_{1}\right) \cap V_{\mathrm{h}}\left(\pi_{2}\right)=\varnothing$, so that $(e, z) \stackrel{M, n}{\Longleftrightarrow}(f, x)$, whence

$$
\frac{\partial \theta_{n}}{\partial p_{e, z}} \leq \alpha_{M}(\mathbf{p}) \frac{\partial \theta_{n}}{\partial p_{f, x}}
$$

by Lemma 13 .

Assume that $H^{\prime} \neq \varnothing$, and write $\left(e^{0}, v^{0}\right)=(e, z)$. We apply the above construction to the triple $\left(e^{0}, v^{0}\right), \pi_{1}, \pi_{2}$ to obtain a family $\left\{\left(e^{0}, v^{0}\right), \sigma_{1}^{1}, \sigma_{2}^{1},\left(e^{1}, v^{1}\right), \pi_{1}^{1}, \pi_{2}^{1}\right\}$. Having constructed the triple $\left(e^{t}, v^{t}\right), \pi_{1}^{t}, \pi_{2}^{t}$ for some $t$, we apply the construction to this triple in order 
to obtain a new family $\left\{\left(e^{t}, v^{t}\right), \sigma_{1}^{t+1}, \sigma_{2}^{t+1},\left(e^{t+1}, v^{t+1}\right), \pi_{1}^{t+1}, \pi_{2}^{t+1}\right\}$, whenever this is possible (i.e., whenever $\left.V_{\mathrm{h}}\left(\pi_{1}^{t}\right) \cap V_{\mathrm{h}}\left(\pi_{2}^{t}\right) \neq \varnothing\right)$. In this way we obtain a sequence $\left(e^{t}, v^{t}\right), \pi_{1}^{t}, \pi_{2}^{t}$, $0 \leq t \leq k$, of triples. The final term is such that $V_{\mathrm{h}}\left(\pi_{1}^{k}\right) \cap V_{\mathrm{h}}\left(\pi_{2}^{k}\right)=\varnothing$. We then set $\left(e^{k+1}, v^{k+1}\right)=(f, x)$.

Each hyperedge $\left(e^{t}, v^{t}\right), 1 \leq t \leq k$, lies in either $\pi_{1}$ or $\pi_{2}$; by Lemma 7 , all hyperedges of $\pi_{1}$ and $\pi_{2}$ are essential, and therefore each $\left(e^{t}, v^{t}\right)$ is essential. Furthermore, by (iii), each $\left(e^{t}, v^{t}\right)$ contains a vertex of $\pi_{1}$; since $V\left(\pi_{1}\right) \subseteq \operatorname{int}(\overline{\Lambda(n)})$, it is the case that $\left(e^{t}, v^{t}\right)$ lies in $\mathbb{E}_{\Lambda(n)}$. Therefore $\left(e^{t}, v^{t}\right) \in \mathcal{E}_{\Lambda(n)}$ for $1 \leq t \leq k$.

Note that the value of $k$ depends generally on the choice of $(e, z),(f, x), M$, and $n$. However, $e^{t}+v^{t} . \kappa \subseteq z . \kappa+\Lambda(M)$ for $0 \leq t \leq k+1$, and the $\left(e^{t}, v^{t}\right)$ are distinct. There are only finitely many hyperedges contained in $z \cdot \kappa+\Lambda(M)$, and, by the translation-invariance of $\mathbb{H}$, we may find a constant $K(M)$, depending on $M$ alone, such that $k \leq K(M)$ for all appropriate $(e, z),(f, x)$, and $n$.

As a consequence of the method of construction of the $\left(e^{t}, v^{t}\right)$, and particularly (i)-(iii) above, we have that

$$
\begin{aligned}
(e, z)=\left(e^{0}, v^{0}\right) & \stackrel{M, n}{\Longleftrightarrow}\left(e^{1}, v^{1}\right) \stackrel{M, n}{\Longleftrightarrow}\left(e^{2}, v^{2}\right) \stackrel{M, n}{\Longleftrightarrow} \cdots \\
& \Longleftrightarrow\left(e^{k}, v^{k}\right) \stackrel{M, n}{\Longleftrightarrow}\left(e^{k+1}, v^{k+1}\right)=(f, x) .
\end{aligned}
$$

We apply Lemma 13 iteratively, to obtain that

$$
\frac{\partial \theta_{n}}{\partial p_{e, z}} \leq \alpha_{M}(\mathbf{p})^{k+1} \frac{\partial \theta_{n}}{\partial p_{f, x}}
$$

The required inequality (6.22) follows from (6.24) and (6.25) by setting

$$
\beta_{M}(\mathbf{p})=\max \left\{1, \alpha_{M}(\mathbf{p})^{K(M)+1}\right\} .
$$

Lemma 14 is the main intermediate step in proving Theorem 2, in the following way. We shall use (6.10) in proving (3.10), in two stages. We partition the set $\mathcal{E}_{\Lambda(n)}$ into two subsets, containing respectively those hyperedges which are 'close' to the boundary of $\overline{\Lambda(n)}$ and those hyperedges which lie in the 'interior' of $\overline{\Lambda(n)}$. For hyperedges $(e, z)$ of the latter type, Lemma 14 may be applied to show that the forthcoming (6.26) is valid for some suitable function $\beta$. Unfortunately, the corresponding argument for hyperedges of the former type is somewhat more complicated, and we deal with such cases later (see Lemmas 16 and 17).

Lemma 15. Let $I=I_{0}$ be given as in Lemma 11. There exists a positive integer $N$ together with a continuous function $\beta:(0,1)^{L} \rightarrow(0, \infty)$, such that the following holds. Let $e, f \in \mathcal{E}$. For all $n \geq N$, and all $z \in \mathbb{Z}^{d}$ such that $z . \kappa+\Lambda(3 I) \subseteq \overline{\Lambda(n)}$,

$$
\frac{\partial \theta_{n}}{\partial p_{e, z}} \leq \beta(\mathbf{p}) \frac{\partial \theta_{n}}{\partial p_{f, z}}
$$

Proof. Suppose $e, f \in \mathcal{E}$, and $z . \kappa+\Lambda(3 I) \subseteq \overline{\Lambda(n)}$. We have from Lemma 11 that $e, f \subseteq$ $\Lambda(I)$, so that $e+z . \kappa, f+z . \kappa \subseteq \overline{\Lambda(n)}$, implying that $(e, z),(f, z) \in \mathcal{E}_{\Lambda(n)}$. Also $e \stackrel{I}{\longrightarrow} f$, which is to say that there exist vertex-disjoint paths $\pi_{1}$ and $\pi_{2}$, joining $e$ to $f$, and using 
hyperedges contained in $\Lambda(I)$. The translated paths $\pi_{1}+z . \kappa, \pi_{2}+z . \kappa$ join $e+z . \kappa$ to $f+z . \kappa$, and use hyperedges contained in $z . \kappa+\Lambda(I)$. We claim that

$$
(z . \kappa+\Lambda(I)) \cap \partial \overline{\Lambda(n)}=\varnothing
$$

from which it follows that $e+z \cdot \kappa \stackrel{I, n}{\longrightarrow} f+z . \kappa$. This in turn implies, by Lemma 14, that (6.26) holds with $\beta=\beta_{I}$ and for all large $n$.

As for the proof of (6.27), suppose that $(z . \kappa+\Lambda(I)) \cap \partial \overline{\Lambda(n)} \neq \varnothing$. There exists (by Lemma 7 and the definition of $\partial \overline{\Lambda(n)})$ an essential hyperedge $(g, y)$ which intersects both $z . \kappa+\Lambda(I)$ and $\overline{\Lambda(n)}^{c}=\mathbb{Z}^{d} \backslash \overline{\Lambda(n)}$. However, by Lemma 11, all essential hyperedges have diameter (in the supremum norm $\|\cdot\|$ ) not exceeding $2 I$, in contradiction of the assumption that $z . \kappa+\Lambda(3 I) \subseteq \overline{\Lambda(n)}$.

Lemma 15 goes most of the way towards proving Theorem 2, by summing (6.26) over all appropriate $z$ and applying (6.10). Unfortunately, a special and non-trivial argument is necessary in order to deal with hyperedges $(e, z)$ which lie near to the boundary of $\overline{\Lambda(n)}$, i.e., those hyperedges $(e, z) \in \mathcal{E}_{\Lambda(n)}$ for which $z . \kappa+\Lambda(3 I) \nsubseteq \overline{\Lambda(n)}$. Intuitively, such a 'boundary effect' should be negligible in comparison with the 'volume effect' provided by Lemma 15. In order to achieve this final step, we introduce a classification of hyperedges in $\mathcal{E}_{\Lambda(n)}$.

Let $\operatorname{int}(\bar{\Lambda})=\bar{\Lambda} \backslash \partial \bar{\Lambda}$. We say that a hyperedge $(e, z)\left(\in \mathcal{E}_{\Lambda(n)}\right)$ is $n$-essential if there exists a path $\pi$ connecting the origin to some vertex of $e+z . \kappa$, and using only vertices of $\operatorname{int}(\overline{\Lambda(n)})$ (if the origin belongs to $e+z . \kappa$, this path may be taken to contain the origin only, assuming that $n$ is such that $0 \in \operatorname{int}(\overline{\Lambda(n)})$ ). Since 0 lies in an essential hyperedge, we have by Lemma 7 that all hyperedges in such a path $\pi$ are essential.

The summation in (6.10) may be restricted to hyperedges $(e, z)$ which are $n$-essential, as part (a) of the following lemma indicates. Part (b) asserts that all hyperedges of $\mathcal{E}_{\Lambda(n)}$ which are sufficiently distant from $\partial \overline{\Lambda(n)}$ are $n$-essential. Part (c) asserts roughly that, if $(e, z)$ is $n$-essential and $R \geq 1$, then there is a path from $e+z . \kappa$ to 0 which, apart from boundedly many vertices lying close to $z$. $\kappa$, uses only vertices contained in $\Lambda(n-R)$.

\section{Lemma 16.}

(a) Let $(e, z) \in \mathcal{E}_{\Lambda(n)}$ and suppose that $(e, z)$ is not n-essential. Then

$$
\frac{\partial \theta_{n}}{\partial p_{e, z}}=0
$$

(b) There exists an absolute constant $J$ such that $(e, z)\left(\in \mathcal{E}_{\Lambda(n)}\right)$ is n-essential for all $e \in \mathcal{E}$ and all $z\left(\in \mathbb{Z}^{d}\right)$ satisfying $z . \kappa \in \Lambda(n-J)$.

(c) For any positive integer $R$, there exists a positive integer $A=A(R)$ such that the following holds. For all sufficiently large $n$, and for all $n$-essential hyperedges $(e, z)$, there exists a path $\pi$ joining a vertex of $e+z . \kappa$ to 0 with $V(\pi) \subseteq \operatorname{int}(\overline{\Lambda(n)})$, and satisfying $V(\pi) \cap \Lambda(n-R)^{c} \subseteq z . \kappa+\Lambda(A)$.

Proof. (a) Suppose that $(e, z)\left(\in \mathcal{E}_{\Lambda(n)}\right)$ is not $n$-essential. Since $(e, z)$ is essential, there exist at least two vertex-disjoint paths joining $e+z . \kappa$ to $\partial \overline{\Lambda(n)}$. Since $(e, z)$ is not $n$ essential, every path of $\mathbb{H}$ joining some vertex of $e+z . \kappa$ to 0 contains some vertex of 
$\partial \overline{\Lambda(n)}$. Hence, in the finite hypergraph obtained from $\mathbb{H}$ by identifying all vertices not in $\operatorname{int}(\overline{\Lambda(n)})$, the composite vertex being labelled $I$, it is the case that all paths from $e+z . \kappa$ to 0 pass through $I$. By Lemma 6 , the event that 0 and $I$ are in the same component is independent of the state of $(e, z)$, whence (6.28) follows.

(b) Let $D$ be as in Lemma 9 , and $I=I_{0}$ as in Lemma 11. Let $J=3 I+D$. Suppose $z . \kappa \in \Lambda(n-J)$ and let $e \in \mathcal{E}$; note that $(e, z) \in \mathcal{E}_{\Lambda(n)}$ since

$$
e+z . \kappa \subseteq z . \kappa+\Lambda(I) \subseteq \Lambda(n-D-2 I) .
$$

There exists a path of $\mathbb{Z}^{d}$ (thought of as a graph with the usual neighbour-relation) joining 0 to some vertex of $e+z . \kappa$ and using only vertices of $\Lambda(n-D-2 I)$. By Lemma 9 , there exists a path $\pi$ of $\mathbb{H}_{\mathcal{E}}$ joining 0 to some vertex of $e+z . \kappa$ using hyperedges contained in $\Lambda(n-2 I-1)$. Since no essential hyperedge has diameter exceeding $2 I$, we have that no vertex of $\pi$ lies in $\partial \overline{\Lambda(n)}$.

(c) For any set $B$ of vertices of $\mathbb{H}$, we let $\partial B$ be the set of vertices $u$ of $B$ for which there exists $v(\notin B)$ such that $u \sim v$. We denote by $\triangle B$ the set of all vertices $v(\notin B)$ with the property that every infinite path of $\mathbb{H}$ beginning at $v$ contains some vertex of $B$. We write $\bar{B}=B \cup \triangle B$.

Let $R, n \geq 1$, and let $(e, z)$ be an $n$-essential hyperedge. For any path $\pi$ joining a vertex of $e+z . \kappa$ to 0 , we define

$$
B(\pi, n)=\min \left\{r: V(\pi) \cap \Lambda(n-R)^{c} \subseteq z . \kappa+\Lambda(r)\right\} .
$$

Thus, $\pi$ uses vertices in the union of $\Lambda(n-R)$ together with the box with radius $B(\pi, n)$ and centre $z . \kappa$. We let

$$
B(e, z, n)=\min _{\pi} B(\pi, n)
$$

where the supremum is taken over all paths $\pi$ joining some vertex of $e+z . \kappa$ to 0 and satisfying $V(\pi) \subseteq \operatorname{int}(\overline{\Lambda(n)})$; such a path exists by the definition of $n$-essentialness. We need to prove that

$$
A=\sup \{B(e, z, n):(e, z) \text { is } n \text {-essential, } n \geq 1\}
$$

satisfies $A<\infty$.

Suppose first that $e+z . \kappa$ contains a vertex of $\Lambda(n-R-D-2 I)$. In this case, by the argument in the proof of part (b), there exists a path $\pi$ joining $e+z . \kappa$ to 0 and using only vertices of $\operatorname{int}(\overline{\Lambda(n)})$ contained in $\Lambda(n-R)$. Such $\pi$ satisfies $V(\pi) \cap \Lambda(n-R)^{c}=\varnothing$, whence

$$
B(e, z, n)=0 \quad \text { if }(e+z . \kappa) \cap \Lambda(n-R-D-2 I) \neq \varnothing .
$$

It follows that we need only consider $n$-essential hyperedges $(e, z)$ satisfying

$$
e+z . \kappa \subseteq \Lambda(n+D) \backslash \Lambda(n-R-D-2 I),
$$

since $\overline{\Lambda(n)} \subseteq \Lambda(n+D)$ by Lemma 9 . The basis of the required argument is the following. The hypergraph $\mathbb{H}$ is invariant under shifts of the form $u \mapsto u+x . \kappa$ for any given $x\left(\in \mathbb{Z}^{d}\right)$, and therefore there are at most $\kappa_{1} \kappa_{2} \cdots \kappa_{d}$ different 'types' of vertex. By (6.30) and (6.31), 
we need only consider vertices within distance $R+D+2 I$ of the bounding hyperplanes of $\Lambda(n)$, i.e., the hyperplanes contributing to $\Lambda(n) \backslash \Lambda(n-1)$. We shall see that there are only finitely many combinations (uniformly in $n$ ) of 'types of vertex' and 'nearby hyperplanes'. In this way, the supremum in (6.29) may be taken over a finite set, implying as required that $A<\infty$.

We do not present the argument in every detail, but there follows sufficient information to check its validity. For a positive integer $n$, we let

$$
H_{i}(n)=\left\{x \in \mathbb{Z}^{d}: x_{i}=n\right\}, \quad L_{i}(n)=\bigcup_{k \leq n} H_{i}(k) .
$$

We define $\overline{L_{i}(n)}$ accordingly and note that, as in the proof of Lemma 9 ,

$$
\overline{L_{i}(n)} \subseteq L_{i}(n+D)
$$

For $v \in \overline{L_{i}(n)}$ we choose a path $\pi=\pi_{i}(v, n)$ of $\mathbb{H}$ such that

(a) $V(\pi) \subseteq \operatorname{int}\left(\overline{L_{i}(n)}\right)=\overline{L_{i}(n)} \backslash \partial \overline{L_{i}(n)}$,

(b) $\pi$ joins $v$ to some vertex of $L_{i}(n-R-D-2 I)$, and

(c) the quantity $r(\pi)$, given by

$$
r(\pi)=\max \{\|x-v\|: x \in V(\pi)\},
$$

is a minimum, subject to (a) and (b) above,

whenever such a path exists. Such a path $\pi_{i}(v, n)$ has vertex set contained in $v+$ $\Lambda\left(r\left(\pi_{i}(v, n)\right)\right)$. We note that $r\left(\pi_{i}(v, n)\right)=0$ if $v \in L_{i}(n-R-D-2 I)$. We denote by $l_{i}(v, n)$ the endpoint of $\pi_{i}(v, n)$ lying in $L_{i}(n-R-D-2 I)$.

Since $\mathbb{H}$ is invariant under shifts of the form $u \mapsto u+x . \kappa$ for any given $x\left(\in \mathbb{Z}^{d}\right)$, there exists (in a manner similar to that of the proof of the final assertion of Lemma 9) an absolute constant $U$ such that

$$
r\left(\pi_{i}(v, n)\right) \leq U
$$

for all triples $(v, n, i)$ for which $\pi_{i}(v, n)$ exists. It follows that

$$
V\left(\pi_{i}(v, n)\right) \subseteq v+\Lambda(U)
$$

whenever $\pi_{i}(v, n)$ exists.

Let $(e, z)$ be an $n$-essential hyperedge of $\mathcal{E}_{\Lambda(n)}$, and assume that (6.31) holds. There exists a vertex $v(\in e+z . \kappa)$ and a path $\pi$ joining $v$ to 0 , satisfying $V(\pi) \subseteq \operatorname{int}(\overline{\Lambda(n)})$. We shall assume that $v=\left(v_{1}, v_{2}, \ldots, v_{d}\right)$ satisfies

$$
v_{i} \geq 0 \text { for all } i
$$

an exactly analogous argument is valid for any other combination of signs of the $v_{i}$, and there are at most $2^{d}$ such combinations. By (6.31), $v$ satisfies

$$
n-R-D-2 I<v_{i} \leq n+D \quad \text { for some } i \in\{1,2, \ldots, d\} .
$$


Since (6.35) holds for some $i$, it holds for some smallest value of $i$, and we assume that

$$
n-R-D-2 I<v_{1} \leq n+D
$$

the following argument is valid for other values of $i$ also.

Suppose, in addition to (6.36), that $v$ satisfies

$$
0 \leq v_{i}<n-R-D-2 I-2 U \text { for } i \in\{2,3, \ldots, d\}
$$

where $U$ satisfies (6.33). Since $(e, z)$ is $n$-essential, and $\operatorname{int}(\overline{\Lambda(n)}) \subseteq \operatorname{int}\left(\overline{L_{1}(n)}\right)$, there exists a path joining $v$ to $L_{1}(n-R-D-2 I)$, using only vertices of int $\left(\overline{L_{1}(n)}\right)$. By $(6.33)-(6.37)$, $\pi_{1}(v, n)$ exists and satisfies

$$
V\left(\pi_{1}(v, n)\right) \subseteq \operatorname{int}(\overline{\Lambda(n)}), \quad l_{1}(v, n) \in \Lambda(n-R-D-2 I) .
$$

By Lemma 9 , there exists a path $\pi^{\prime}$ joining $l_{1}(v, n)$ to 0 , and such that $V\left(\pi^{\prime}\right) \subseteq$ $\Lambda(n-R) \cap \operatorname{int}(\overline{\Lambda(n)})$. The union of $\pi_{1}(v, n)$ and $\pi^{\prime}$ contains a path joining $v$ to 0 , using vertices of $\operatorname{int}(\overline{\Lambda(n)})$ which belong to $\Lambda(n-R) \cup\{v+\Lambda(U)\}$. Now

$$
v \in e+z . \kappa \subseteq z . \kappa+\Lambda(I) .
$$

Therefore $\|v-z . \kappa\| \leq I$, implying that

$$
B(e, z, n) \leq U+I
$$

under the assumptions (6.36) and (6.37).

Suppose now that (6.36) holds but that (6.37) fails to hold for some $i(\geq 2)$. Then (6.37) fails for some smallest value of $i$, and we shall assume for simplicity of notation that this value is $i=2$; an exactly analogous argument is valid for other values. Assume then that

$$
n-R-D-2 I<v_{1} \leq n+D, \quad n-R-D-2 I-2 U \leq v_{2} \leq n+D .
$$

In the argument above (from (6.32) onwards) we replace $H_{1}(n)$ and $L_{1}(n)$ by

$$
\begin{aligned}
& H_{12}(n)=\left\{x \in \mathbb{Z}^{d}: x_{1}=n, x_{2} \leq n\right\} \cup\left\{x \in \mathbb{Z}^{d}: x_{1} \leq n, x_{2}=n\right\}, \\
& L_{12}(n)=\bigcup_{k \leq n} H_{12}(k)=L_{1}(n) \cap L_{2}(n),
\end{aligned}
$$

and we define $\overline{L_{12}(n)}$ accordingly. For $v \in \overline{L_{12}(n)}$ we find a path $\pi=\pi_{12}(v, n)$ which

(a) uses only vertices of $\operatorname{int}\left(\overline{L_{12}(n)}\right)=\overline{L_{12}(n)} \backslash \partial \overline{L_{12}(n)}$,

(b) joins $v$ to some vertex in $L_{12}(n-R-D-2 I)$,

(c) is such that $r(\pi)$ is a minimum subject to (a) and (b),

whenever such a path exists. We write $l_{12}(v, n)$ for the endvertex of $\pi_{12}(v, n)$ lying in $L_{12}(n-R-D-2 I)$.

As before, there exists an absolute constant $U_{12}$ such that

$$
r\left(\pi_{12}(v, n)\right) \leq U_{12} \quad \text { whenever } \pi_{12}(v, n) \text { exists. }
$$


Assume in addition to (6.38) that

$$
0 \leq v_{i}<n-R-D-2 I-2\left(U+U_{12}\right) \text { for } i \geq 3
$$

Under this extra assumption, it is the case that

(a) $\pi_{12}(v, n)$ exists,

(b) $V\left(\pi_{12}(v, n)\right) \subseteq \operatorname{int}(\overline{\Lambda(n)})$,

(c) $l_{12}(v, n) \in \Lambda(n-R-D-2 I)$.

There exists a path $\pi^{\prime}$ joining $l_{12}(v, n)$ to 0 , and using only vertices of $\operatorname{int}(\overline{\Lambda(n)})$ contained in $\Lambda(n-R)$. The union of $\pi_{12}(v, n)$ and $\pi^{\prime}$ contains a path $\pi$ joining $v$ to 0 , using vertices of $\operatorname{int}(\overline{\Lambda(n)})$ which belong to $\Lambda(n-R) \cup\left\{v+\Lambda\left(\max \left\{U, U_{12}\right\}\right)\right\}$. It follows as before that $B(e, z, n) \leq \max \left\{U, U_{12}\right\}+I$ under assumptions (6.38) and (6.40).

We now iterate this argument. At the next stage, we suppose that (6.40) fails for some $i(\geq 3)$, say the value $i=3$. That is, we replace (6.38) by

$$
\begin{gathered}
n-R-D-2 I<v_{1} \leq n+D, \quad n-R-D-2 I-2 U \leq v_{2} \leq n+D, \\
n-R-D-2 I-2\left(U+U_{12}\right) \leq v_{3} \leq n+D .
\end{gathered}
$$

Arguing as above, but replacing $H_{12}(n)$ by

$$
H_{123}(n)=\left\{x \in \mathbb{Z}^{d}: x_{1}, x_{2}, x_{3} \leq n, x_{i}=n \text { for some } i=1,2,3\right\},
$$

and making further appropriate changes, we obtain $B(e, z, n) \leq \max \left\{U, U_{12}, U_{123}\right\}+I$ for some appropriate constant $U_{123}$, whenever it is the case that

$$
0 \leq v_{i}<n-R-D-2 I-2\left(U+U_{12}+U_{123}\right) \quad \text { for } i \geq 4
$$

in place of $(6.40)$.

We may continue in like manner until all of the (bounded number of) possible cases have been dealt with. We have made various assumptions above, particularly in the choices of $i$ in (6.35), (6.38), and (6.41). At each of these (and subsequent) stages, there are only boundedly many possibilities (uniformly in all large $n$ ), and the corresponding argument is valid for all of them. Since each possibility leads to the conclusion that $B(e, z, n)$ is uniformly bounded for all large $n$, we deduce that

$$
B(e, z, n) \leq U^{\prime} \quad \text { if } v \geq(0,0, \ldots, 0)
$$

for some absolute constant $U^{\prime}$, so long as $n$ is sufficiently large. Exactly similar arguments are valid when some of the $v_{i}$ are negative. Since there are only boundedly many combinations of signs of the $v_{i}$, we deduce that the constant $A$ given in (6.29) is finite, and the proof is complete.

With the aid of Lemma 16, we may establish the final step in the proof of Theorem 2.

Lemma 17. Let $I=I_{0}$ be given as in Lemma 11. There exist positive integers $R, S$, and a continuous function $\gamma:(0,1)^{L} \rightarrow(0, \infty)$, such that the following holds. Let $e, f \in \mathcal{E}$, and let $n \geq R$. Suppose that $z$ is such that

$$
z . \kappa+\Lambda(3 I) \nsubseteq \overline{\Lambda(n)}, \text { and }(e, z) \text { is n-essential. }
$$


There exists $y \in \mathbb{Z}^{d}$ such that $\|y-z\| \leq S,(f, y)$ is n-essential, and

$$
\frac{\partial \theta_{n}}{\partial p_{e, z}} \leq \gamma(\mathbf{p}) \frac{\partial \theta_{n}}{\partial p_{f, y}}
$$

Before proving Lemma 17, we show how it may be used to deduce Theorem 2. Let $I, N, R, S, \beta, \gamma$ be as in Lemmas 15 and 17 , and suppose $n \geq \max \{N, R\}$. Fix $e, f \in \mathcal{E}$. Let $Z_{1}$ be the set of all $z\left(\in \mathbb{Z}^{d}\right)$ such that $z . \kappa+\Lambda(3 I) \subseteq \overline{\Lambda(n)}$, and let $Z_{2}$ be the set of all $z$ such that $(e, z) \in \mathcal{E}_{\Lambda(n)}$ and $z . \kappa+\Lambda(3 I) \nsubseteq \overline{\Lambda(n)}$. We have by (6.26) that

$$
\sum_{z \in Z_{1}} \frac{\partial \theta_{n}}{\partial p_{e, z}} \leq \beta(\mathbf{p}) \sum_{z \in Z_{1}} \frac{\partial \theta_{n}}{\partial p_{f, z}} .
$$

Furthermore, by Lemmas 16(a) and 17,

$$
\sum_{z \in Z_{2}} \frac{\partial \theta_{n}}{\partial p_{e, z}}=\sum_{\substack{z \in Z_{2}: \\(e, z) \text { is } n \text {-essential }}} \frac{\partial \theta_{n}}{\partial p_{e, z}} \leq \gamma(\mathbf{p}) \sum_{z \in Z_{2}} \frac{\partial \theta_{n}}{\partial p_{f, y}}
$$

where $y=y(e, f, z, n)$ satisfies $\|y-z\| \leq S$, and $(f, y)$ is $n$-essential. Let $T$ be an upper bound for the number of points $x\left(\in \mathbb{Z}^{d}\right)$ satisfying $\|x\| \leq S$. By (6.46),

$$
\sum_{z \in Z_{2}} \frac{\partial \theta_{n}}{\partial p_{e, z}} \leq T \gamma(\mathbf{p}) \sum_{y:(f, y) \in \mathcal{E}_{\Lambda(n)}} \frac{\partial \theta_{n}}{\partial p_{f, y}},
$$

which may be combined with (6.45) to yield, via (6.10),

$$
\frac{\partial \theta_{n}}{\partial p_{e}} \leq \alpha(\mathbf{p}) \frac{\partial \theta_{n}}{\partial p_{f}}
$$

where $\alpha(\mathbf{p})=\beta(\mathbf{p})+T \gamma(\mathbf{p})$. This proves Theorem 2 .

Proof of Lemma 1\%. Let $n \geq 1, e, f \in \mathcal{E}$, and let $(e, z)$ satisfy (6.43). Let $D$ be given as in Lemma $9, I_{x}$ as in Lemma 11 , and $J$ as in Lemma 16 (b). For all sufficiently large $n$, we may find $y\left(\in \mathbb{Z}^{d}\right)$ such that

(a) $\|y-z\| \leq 2(3 I+J+D)$,

(b) $y . \kappa \in \Lambda(n-J)$, and

(c) $e+z . \kappa$ and $f+y . \kappa$ are disjoint,

where $I=I_{0}$; we choose $y$ accordingly. We have from Lemma $16(\mathrm{~b})$ that $(f, y)$ is $n$ essential. Using Lemma 11,

$$
e \stackrel{T}{\longrightarrow} f+(y-z) \cdot \kappa
$$

where $T=\max \left\{I_{u}:\|u\| \leq 2(3 I+J+D)\right\}$. Therefore there exist at least two vertexdisjoint paths $\pi_{1}, \pi_{2}$ joining vertices of $e+z . \kappa$ to vertices of $f+y . \kappa$, these paths using hyperedges contained within $z . \kappa+\Lambda(T)$. Either $(e, z) \stackrel{T, n}{\longrightarrow}(f, y)$, or not. If $(e, z) \stackrel{T, n}{\longrightarrow}(f, y)$, we may apply Lemma 14 to find that

$$
\frac{\partial \theta_{n}}{\partial p_{e, z}} \leq \beta_{T}(\mathbf{p}) \frac{\partial \theta_{n}}{\partial p_{f, y}}
$$


for all large $n$, in which case (6.44) is proved for suitable $\gamma$ and $S$.

Suppose then that it is not the case that $(e, z) \stackrel{T, n}{\longrightarrow}(f, y)$. In this case, we must pick the above pair $\pi_{1}, \pi_{2}$ in such a way that both $\pi_{1}$ and $\pi_{2}$ contain vertices lying in $\partial \overline{\Lambda(n)}$. For $i=1,2$, we write $\pi_{i}(e, z)$ (respectively $\pi_{i}(f, y)$ ) for the maximal subpath of $\pi_{i}$ containing the endvertex of $\pi_{i}$ lying in $e+z . \kappa$ (respectively, lying in $\left.f+y . \kappa\right)$ but containing no vertex of $\partial \overline{\Lambda(n)}$.

Since $(e, z)$ (respectively $(f, y))$ is $n$-essential, there exists a path $\pi_{e}$ (respectively $\pi_{f}$ ) joining some vertex of $e+z . \kappa$ (respectively $f+y . \kappa)$ to 0 , and using only vertices of int $(\overline{\Lambda(n)})$. By Lemma 16(c) and assumption (a) above, we may choose $\pi_{e}$ and $\pi_{f}$ with the following property: there exists an absolute constant $A=A(D, I, J)$ such that any vertex $v$ of $\pi_{e}$ or $\pi_{f}$ which is not contained in $\Lambda(n-2 D-2 I)$ satisfies $v \in z . \kappa+\Lambda(A)$. By appealing to Lemma 9 , we may find a constant $B=B(A, I, D, T)$ such that there exists a path $\pi_{e f}$ joining some vertex of $e+z . \kappa$ to some vertex of $f+y . \kappa$, and using only vertices in $\operatorname{int}(\overline{\Lambda(n)}) \cap(z . \kappa+\Lambda(B)) ; c f .(6.27)$. We may assume that $B \geq T$.

If $\pi_{e f}$ uses no vertex contained in either $\pi_{1}$ or $\pi_{2}$ (except possibly for vertices of $e+z . \kappa$ or $f+y . \kappa)$, then

$$
(e, z) \stackrel{B, n}{\longrightarrow}(f, y),
$$

whence (6.44) follows by Lemma 14 for appropriate $\gamma$ and $S$; see Figure 5. On the other hand, if $\pi_{e f}$ uses some such vertex, then there may be a last vertex of $\pi_{e f}$ (proceeding along $\pi_{e f}$, starting from its endpoint in $\left.e+z . \kappa\right)$ lying on either $\pi_{1}(e, z)$ or $\pi_{2}(e, z)$, and there may be a first subsequent vertex lying on either $\pi_{1}(f, y)$ or $\pi_{2}(f, y)$. More precisely, let $w_{1}, w_{2}, \ldots, w_{r}$ be the vertices of $\pi_{e f}$ in order, where $w_{1} \in e+z . \kappa$ and $w_{r} \in f+y . \kappa$. Let

$$
\begin{aligned}
& i=\max \left\{k: w_{k} \in V\left(\pi_{1}(e, z)\right) \cup V\left(\pi_{2}(e, z)\right)\right\}, \\
& j=\min \left\{k: k>i, w_{k} \in V\left(\pi_{1}(f, y)\right) \cup V\left(\pi_{2}(f, y)\right)\right\},
\end{aligned}
$$

with the convention that the maximum (respectively minimum) of the empty set is $-\infty$ (respectively $\infty$ ). Suppose that $1 \leq i, j \leq r$, and we may suppose without loss of generality that $w_{i}$ lies on $\pi_{1}(e, z)$, and $w_{j}$ lies on $\pi_{l}(f, y)$ for some $l$. In such a case, one may see as follows that (6.47) holds. One path from $e+z . \kappa$ to $f+y . \kappa$ of the required form is obtained by following $\pi_{1}(e, z)$ to $w_{i}$, then following $\pi_{e f}$ from $w_{i}$ to $w_{j}$, and thence following $\pi_{l}(f, y)$ to $f+y . \kappa$; if this sequence does not form a path, then a path may be obtained from it by a process of loop-removal. Another such path is obtained by following $\pi_{2}(e, z)$ to $\partial \overline{\Lambda(n)}$, then some path of $\mathbb{Z}^{d} \backslash \operatorname{int}(\overline{\Lambda(n)})$ to a vertex of $\partial \overline{\Lambda(n)}$ adjacent to an endpoint of $\pi_{k}(f, y)$ (where $k \neq l$ ), then following $\pi_{k}(f, y)$ to $f+y . \kappa$. These two paths are vertex-disjoint, and we have in conclusion that (6.47) holds as before.

The remaining two cases arise when either $i=-\infty$ or $j=\infty$; certainly, either $i \geq 1$ or $j \leq r$, by the assumption that $\pi_{e f}$ has some vertex in common with $V\left(\pi_{1}\right) \cup V\left(\pi_{2}\right)$. Suppose that $i=-\infty$ and $j \leq r$ (the other case is similar). One path of the required sort is obtained by following $\pi_{e f}$ from $w_{1}$ to $w_{j}$, then $\pi_{l}(f, y)$ from $w_{j}$ to $f+y . \kappa$; the other such path is obtained as before. Thus (6.47) holds.

Proof of Theorem 3. (a) If $\widehat{\mathbf{p}}(\mathbf{J}, \beta)=\widehat{\mathbf{p}}\left(\mathbf{J}^{\prime}, \beta\right)$ for all $\beta$ then, by (3.8),

$$
\theta(\mathbf{p}(\mathbf{J}, \beta), q)=\theta\left(\mathbf{p}\left(\mathbf{J}^{\prime}, \beta\right), q\right) \quad \text { for all } \beta,
$$


whence $\beta_{c}(\mathbf{J}, q)=\beta_{c}\left(\mathbf{J}^{\prime}, q\right)$.

(b) Certainly $\beta_{c}(\cdot, q)$ is non-increasing, since $\theta(\cdot, q)$ is non-decreasing. Suppose it is the case that $\beta_{c}(\mathbf{J}, q)=\beta_{c}\left(\mathbf{J}^{\prime}, q\right)\left(=\beta_{c}\right.$, say) for some pair $\mathbf{J}, \mathbf{J}^{\prime}$ satisfying the conditions of the theorem. Then $\mathbf{p}\left(\mathbf{J}, \beta_{c}\right), \mathbf{p}\left(\mathbf{J}^{\prime}, \beta_{c}\right) \in \mathrm{C}_{q}$, in contradiction of Theorem 1(b), particularly the deduction at the end of the subsequent paragraph.

Acknowledgements. This work was begun during a visit to Cornell University, with partial support from the U.S. Army Research Office through the Mathematical Sciences Institute of Cornell University. The author benefited from conversations with Carol Bezuidenhout and Harry Kesten. 


\section{REFERENCES}

Aizenman, M., Chayes, J. T., Chayes, L., and Newman, C. M. (1988). Discontinuity of the magnetization in one-dimensional $1 /|x-y|^{2}$ Ising and Potts models. Journal of Statistical Physics 50, 1-40.

Aizenman, M. and Grimmett, G. R. (1991). Strict monotonicity for critical points in percolation and ferromagnetic models. Journal of Statistical Physics 63, 817-835.

Barlow, R. N. and Proschan, F. (1965). Mathematical Theory of Reliability. Wiley, New York.

Berge, C. (1973). Graphs and Hypergraphs. North-Holland, London.

Bezuidenhout, C. E., Grimmett, G. R., and Kesten, H. (1993), [BGK]. Strict inequality for critical values of Potts models and random-cluster processes. Communications in Mathematical Physics 158, 1-16.

Edwards, R. G. and Sokal, A. D. (1988). Generalization of the Fortuin-KasteleynSwendsen-Wang representation and Monte Carlo algorithm. Physical Review D 38, 2009-2012.

Grimmett, G. R. (1989). Percolation. Springer-Verlag, Berlin.

Grimmett, G. R. (1993). Differential inequalities for Potts and random-cluster processes. In Cellular Automata and Cooperative Systems, ed. N. Boccara et al., Kluwer, Dordrecht, $227-236$.

Grimmett, G. R. (1994). Percolative problems. In Probability and Phase Transition, ed. G. Grimmett, Kluwer, Dordrecht, 69-86, to appear.

Hammersley, J. M. (1961). Comparison of atom and bond percolation. Journal of Mathematical Physics 2, 728-733.

Holley, R. (1974). Remarks on the FKG inequalities. Communications in Mathematical Physics 36, 227-231.

Kesten, H. (1982). Percolation Theory for Mathematicians. Birkhäuser, Boston.

Menshikov, M. (1987). Quantitative estimates and rigorous inequalities for critical points of a graph and its subgraphs. Theory of Probability and its Applications 32, 544-547.

Wierman, J. C. (1993). Equality of directional critical exponents in multiparameter percolation models. In preparation.

Statistical Laboratory,

Department of Pure Mathematics and Mathematical Statistics,

University of Cambridge,

16 Mill Lane,

Cambridge CB2 1SB, UK 


\section{FIGURE CAPTIONS}

Figure 1. The upper pair depict the interacting sets $e_{1}$ and $e_{2}$ of Example 1 (in dotted lines, and a solid line, respectively, on the left), together with the associated lattice $G_{1}$. The lower pair depict $f_{1}$ and $f_{2}$ in Example 2, and the lattice $G_{2}$.

Figure 2. Every infinite path beginning in $e+z . \kappa$ passes through each $c_{i}$. The set $g(e, z)$ comprises all vertices to the left of $c(e, z)$ in this diagram.

Figure 3. The hyperedges $(e, z)$ and $(f, x)$ are joined by two paths $\pi_{1}$ and $\pi_{2}$, such that $\pi_{1}$ uses vertices of int $(\overline{\Lambda(n)})$ only, and $V_{\mathrm{h}}\left(\pi_{1}\right) \cap V_{\mathrm{h}}\left(\pi_{2}\right)=\varnothing$. No other hyperedges of $\mathbb{E}_{\Lambda(n)}$, lying in $B_{z}$, are present. The smaller box is $z . \kappa+\Lambda(M)$.

Figure 4. Cases $\mathrm{A}-\mathrm{E}$ in the definition of the family $\left\{(h, x), \sigma_{1}, \sigma_{2},\left(h^{\prime}, x^{\prime}\right), \pi_{1}^{\prime}, \pi_{2}^{\prime}\right\}$. In each case, the two leftmost lines depict $\sigma_{1}$ and $\sigma_{2}$, and the two rightmost lines depict $\pi_{1}^{\prime}$ and $\pi_{2}^{\prime}$. The left-hand endpoint of each $\pi_{i}$ lies in $h+x . \kappa$; the value of $\left(h^{\prime}, x^{\prime}\right)$ is given in the right-hand column. The rectangles depict $e_{1}$ and $e_{2}$. In Case $\mathrm{C}$, the new vertex $w$ lies in both $e_{1}$ and $e_{2}$.

Figure 5. A sketch of the situation in the proof of Lemma 17, when $\pi_{e f}$ has no vertices in common with $\pi_{1}$ and $\pi_{2}$. 